This is the pre-peer reviewed version of the following article: Jordana K. Palacios, Heng Zhang, Bin Zhang, Nikos Hadjichristidis, Alejandro J. Müller, Direct identification of three crystalline phases in PEO- $b$-PCL- $b$ PLLA triblock terpolymer by In situ hot-stage atomic force microscopy, Polymer 205 : (2020) // Article ID 122863, which has been published in final form at https://doi.org/10.1016/j.polymer.2020.122863. (C) 2020 Elsevier. This article may be used for non-commercial purposes in accordance with Elsevier Terms and Conditions for Use of Self-Archived Versions

\title{
Direct Identification of Three Crystalline Phases in PEO-b-PCL-b-PLLA Triblock Terpolymer by In Situ Hot-Stage Atomic Force Microscopy
}

\author{
Jordana K. Palacios ${ }^{1-2}$, Heng Zhang ${ }^{3}$, Bin Zhang ${ }^{3 *}$, Nikos Hadjichristidis ${ }^{4 *}$, \\ Alejandro J. Müller ${ }^{1,5 *}$
}

${ }^{1}$ POLYMAT and Polymer Science and Technology Department, Faculty of Chemistry, University of the Basque Country UPV/EHU, Paseo Manuel de Lardizabal 3, 20018 Donostia-San Sebastián, Spain.

${ }^{2}$ Fundación Centro Tecnológico Miranda de Ebro (CTME), R\&D Materials Department, Miranda de Ebro, 09200, Burgos, Spain.

${ }^{3}$ School of Materials Science \& Engineering, Zhengzhou University, Zhengzhou 450002, People’s Republic of China.

${ }^{4}$ King Abdullah University of Science and Technology (KAUST), Physical Sciences and Engineering Division, KAUST Catalysis Center, Thuwal, Saudi Arabia.

${ }^{5}$ IKERBASQUE, Basque Foundation for Science, Bilbao, Spain.

*Corresponding authors: $\quad$ binzhang@zzu.edu.cn, $\quad$ Nikolaos.Hadjichristidis@kaust.edu.sa, alejandrojesus.muller@ehu.es 


\section{ABSTRACT}

In this work, we provide a detailed description of the tri-lamellar nanoscale morphology of a triple crystalline PEO- $b$-PCL- $b$-PLLA triblock terpolymer obtained by HotStage Atomic Force microscopy (AFM) imaging and Wide Angle X-ray scattering (WAXS) analysis for the first time. The precursor PCL- $b$-PLLA diblock copolymer has also been included in the study for comparison purposes. A two-step crystallization protocol has been applied to create a distinct lamellar morphology. Both WAXS and AFM revealed the double crystalline nature of the diblock copolymer. However, the identification of multiple crystalline phases in the triblock terpolymer by AFM and WAXS at room temperature is not straightforward. The advantages of hot-stage AFM allowed following the evolution of the lamellar morphology and the successive melting of the tricrystalline PEO- $b$-PCL- $b$-PLLA sample during heating. Taking into account the melting temperature of each crystalline block, the existing lamellar populations were clearly identified. At $45^{\circ} \mathrm{C}$, the thinnest lamellae disappeared, due to the melting of PEO crystals. The medium size lamellae disappeared at $60{ }^{\circ} \mathrm{C}$ when PCL crystals melt. At that temperature, the only remaining crystals are those of the PLLA block. AFM mechanical modulus images provide further evidence of the lamellar self-assembly of the triblock terpolymer. The nanoscale arrangement includes lamellae of PCL, PEO, or both in between the PLLA lamellae. Hot-Stage AFM is a valuable technique to elucidate the morphological features of complex multi-crystalline systems.

Keywords: PEO- $b$-PCL- $b$-PLLA triblock terpolymer; tri-lamellar morphology; Hot-Stage AFM; WAXS 


\section{INTRODUCTION}

The morphology of block copolymers has attracted broad interest in the polymer scientific community in the last decade. The micro and nanostructural features are influenced by several factors, such as melt miscibility, crystallinity, composition, and thermal conditioning (i.e., crystallization, annealing, and thermal history in general). A wide range of different morphologies can be developed (such as lamellae, spheres, cylinders, 2D and 3D aggregates) that depend on whether the block copolymer is miscible or melt segregated, or if it is amorphous, semicrystalline or combined (double crystalline, double amorphous, crystalline-amorphous, etc.). ${ }^{1-7}$

To study the morphology of block copolymers in real space, a series of techniques can be used, such as polarized light optical microscopy (PLOM), transmission electron microscopy (TEM), and atomic force microscopy (AFM). Among them, AFM is an imaging technique of high-resolution that not only substantially complements the interpretation of TEM and PLOM observations but also of other structural characterization techniques in reciprocal space, such as X-ray diffraction. AFM allows direct visualization of the nanoscale structure. Besides, one of the advantages of AFM is sample preparation. Unlike TEM, AFM does not require thin sectioning, etching, or staining. However, it should be kept in mind that AFM is a surface technique, and it might not represent bulk structural behavior. AFM applications in polymers include the study of polymer morphologies at micro and nanoscale level, the identification of molecular order in single crystals and oriented samples, individual polymer chains and branches, filled polymer systems and composites, polymer blends and block copolymers. ${ }^{8-10}$ 
AFM has been used to detect microphase-separated morphologies of block copolymers. Block copolymers display different morphologies depending on three main factors: copolymer composition and architecture, melt segregation strength, and thermal transitions (e.g., order-disorder, crystallization, and glass transition temperatures). ${ }^{3,5}$ The ordered superstructure sizes range from micro to nanoscale level. For instance, AFM has been useful to detect several types of microdomain patterns in strongly segregated systems, such as spheres, “worm”-like, lamellae, etc., as well as, lamellar nanodomains in weakly segregated or melt miscible systems. Particularly, melt miscible (or weakly segregated) block copolymers with crystallizable blocks, the final morphology is a consequence of the crystallization conditions and the microphase segregation driven by the crystallization event. Employing suitable crystallization conditions, AFM examinations at room temperature reveal the self-assembly of the polymer chains into mixed axialitic or spherulitic-type superstructures composed of lamellar arrangements., ${ }^{2,}$, 7, 11-28

A group of well-investigated melt miscible (or weakly segregated) double crystalline systems are the diblock and triblock copolymers composed of poly(L-lactide) (PLLA), poly(caprolactone) (PCL) and poly(ethylene oxide) (PEO). These diblock copolymers are of high interest because of their good physical properties and biodegradability. Extensive research has been published regarding the microscale morphology of these diblock copolymers and their corresponding homopolymers. Spherulites, banded or concentric spherulites, axialities, 2D aggregates, among others crystalline textures, have been reported. $24,27,29-33$

For a few years, we have been investigating the complexity of the morphology and crystallization of unique $\mathrm{ABC}$ triblock terpolymers, in which the three blocks are able to 
crystallize when the length of the blocks and the crystallization conditions are adjusted properly. To that purpose, model triblock terpolymers of PLLA, PCL, and PEO blocks (PEO$b$-PCL-b-PLLA) have been exhaustively studied., ${ }^{43-36}$ Particularly, the morphology of these melt miscible and triple crystalline triblock terpolymers have been reported by Chiang et al. ${ }^{37}$ and Palacios et al. ${ }^{33,35,36}$ Wide-angle X-Ray scattering (WAXS) measurements, carried out during cooling, confirmed that the PLLA block is the one that crystallizes first, followed by the PCL and lastly, the PEO. PLOM observations indicated that the microscale structure is templated by the PLLA block as a result of its crystallization. The successive crystallization of the PCL and PEO blocks does not change the microscale superstructure templated by the PLLA block. The evidence of the crystallization of the other two blocks is that the magnitude of the birefringence varies. ${ }^{33}$ Linear and cyclic diblock copolymers including PCL, PLLA and PEO also exhibit this behavior. ${ }^{11,31,32,38-43}$ Chiang et al. ${ }^{37}$ presented single crystals of PEO-b-PCL-b-PLLA triblock terpolymers crystallized from solution A sequential layer-bylayer crystallization was observed by modifying the crystallization conditions (e.g., polymersolvent interaction, fuming time).

Some features of the triple crystalline nanoscale morphology of PEO-b-PCL- $b$-PLLA triblock terpolymers have been reported by some of us in a previous publication. ${ }^{35}$ Polymer sample films were crystallized from melt, and the final morphology was observed by AFM at room temperature. A tri-lamellar self-assembly that included lamellae of the three phases was indirectly elucidated from AFM observations complemented with SAXS experiments and theoretical simulations. From our observations, we proposed a lamellar arrangement that includes the alternation of only one lamella of either PCL or PEO in between two lamellae of PLLA. However, a clear identification of such peculiar variation of the lamellar crystalline 
phases was not possible by only room temperature AFM observations. Therefore, the next step would be to apply a thermal scan to the lamellar morphology observed at room temperature to get further insight into the tri-lamellar structure and long-range order.

AFM advantages include that the measurements can be extended from room to higher temperatures. Prilliman et al. ${ }^{44}$ reported in 1998 the development of a Hot-Stage AFM in tapping mode. A coupled cooling/heating device allows in situ visualization of the morphological changes resulting from thermal transitions. The possibility of imaging phase thermal transitions is a valuable analysis that complements other thermal characterization techniques such as differential scanning calorimetry (DSC) and dynamic mechanical analysis (DMA). To study phase transitions, images at different temperatures are recorded after the sample has been heated or cooled, or the AFM probe is heated and is kept in constant contact with the sample during the heating scan or during the phase transition. As the probe temperature exceeds the thermal transition, the polymeric material under it can be recognized. ${ }^{8,9}$

More recently, Zhang et $a .^{45}$ reported a very innovative approach for an AFM heating/cooling device. The concept is highly useful to in situ apply a thermal treatment to a sample, and then, observe it with the AFM. The authors coupled a sensor for fast scanning chip calorimetry (FSC) to the scanner head of a non-contact mode AFM. The sensor includes the sample, and it is placed under the AFM probe tip for scanning. This sensor is simultaneously connected to the electronic of an FSC device. The FSC technique can perform DSC scans at extremely fast cooling and heating rates (up to $1.000 .000 \mathrm{~K} / \mathrm{s}$ ) and apply isothermal treatments to a sample. In this way, a particular morphology can be induced under specific conditions. Then, the morphology created can be observed by AFM at that 
temperature, or it can be "frozen” by quenching the sample rapidly to room temperature (or lower) and then observed it. The novel approach represents a Fast Hot-Stage for AFM that simultaneously provides DSC information of the sample (crystallinity, thermal transitions, etc.).

AFM has already been used to image the melting and crystalline morphology during crystallization at high temperatures for some semicrystalline polymers. Some examples are the reports published on polyethylene (PE), ${ }^{8,46-48}$ PCL, ${ }^{49-51}$ and PEO. ${ }^{52-55}$ Additionally, hotstage AFM has been used to examine polymer blends and block copolymers. Early studies were performed in PEO/poly(methyl methacrylate) blends by Pearce et al. in 1998. The authors followed the crystallization of the PEO and observed the lamellar growth and the impingement of adjacent spherulites. Wang et al. ${ }^{56}$ also reported the morphological changes during crystallization in blends of PEO and poly(butylene succinate) (PBS) with different compositions. The authors employed hot-stage AFM to examine the crystallizability of the blends and observed that the isothermal crystallization temperature is chosen for the PBS phase and the composition, both had an important effect on PEO crystallization and on the local distribution of the PEO crystals. The PEO crystalline phase can locate at the interlamellar spaces of the PBS crystals or can grow on the edges of the PBS lamellae.

Imaging semicrystalline diblock copolymers at high temperatures, employing hotstage AFM, should provide more insight into the lamellar arrangement at the nanoscale. However, only a few reports on single and double crystalline diblock copolymers have been published, and most of the AFM polymer crystal observations deal with samples crystallized from solution and only a few with samples crystallized from the melt. In diblock copolymers of PEO containing a tablet-like block of poly(2,5-bis[(4- 
methoxyphenyl)oxycarbonyl]styrene) (PMPCS) (PEO-b-PMPCS), ${ }^{57}$ hot-stage AFM has been used to follow the PEO block crystallization. Crystallizing ultrathin films from the melt usually produces flat-on lamellae with chain direction perpendicular to the substrates. Under isothermal conditions, the PEO crystallized into dendritic structures if the crystallization temperature is lower than $44{ }^{\circ} \mathrm{C}$, but the morphology changed to square-shaped crystals at temperatures higher than $48{ }^{\circ} \mathrm{C}$. The crystals were completely melted at $56{ }^{\circ} \mathrm{C}$ or temperatures above this one. By AFM, the authors were also able to determine that the lamellar thickness increased as the crystallization temperature was higher. ${ }^{57}$ Hot-stage AFM has also been used by Zhan et al. ${ }^{58}$ to examine the relief structure of PS-b-PCL diblock copolymers. In these copolymers, the PCL block only crystallized, and the AFM technique was employed to analyze the coupling and complex competition between dewetting and microphase separation.

To our knowledge, only Cui et al. ${ }^{59}$ have followed the structural changes upon heating and cooling in double crystalline PCL-b-PLLA diblock copolymers employing hot-stage AFM. The authors studied the PLLA block crystallization behavior upon cooling from melt and upon heating from room temperature with AFM. Different crystalline morphologies were observed depending on the crystallization conditions. However, there was not a clear distinction between the PCL and PLLA lamellae.

In this paper, we take advantage of the Hot-Stage AFM approach to provide a clear elucidation of the trilayered lamellar morphology of an ABC-type triple crystalline triblock terpolymer. To our knowledge, this is the first time that Hot-Stage AFM is employed to identify three different crystalline phases successfully in the same polymeric sample. The PEO- $b$-PCL- $b$-PLLA is a triblock terpolymer with an alternating superstructure of three 
crystalline phases, as we had reported in previous publications. ${ }^{43}{ }^{33-36}$ The PLLA block crystallizes first and produces a spherutilic template where the PCL and PEO blocks have to crystallize. Here, we report the in situ hot-stage AFM observations of the sequential melting of isothermally crystallized model PEO- $b$-PCL- $b$-PLLA triblock terpolymer films. Complementary WAXS analysis is provided to support the AFM evidence. Assessing the distribution of discrete crystals provides a deeper understanding of the sequential crystallization and melting in PEO- $b$-PCL- $b$-PLLA triblock terpolymers.

\section{EXPERIMENTAL PART}

\section{Materials}

A diblock copolymer precursor (PCL- $b$-PLLA) and a PEO- $b$-PCL- $b$-PLLA triblock terpolymer were selected for the morphological characterization. These materials were synthesized as previously reported by a one-pot sequential organocatalytic ring-opening sequential polymerization of ethylene oxide, $\varepsilon$-caprolactone, and L-lactide. A phosphazene base, 1-tert-butyl-2,2,4,4,4-pentakis-(dimethylamino)-2 $\lambda 5,4 \lambda 5$-catenadi(phosphazene) (tBuP2) was employed as a single catalyst. For more details on the synthesis procedure and characterization, refer to ${ }^{60,61}$, and the references in them. The PLLA and PCL block length in both terpolymer and diblock copolymer was very similar (see Table 1) to avoid any influence of molecular weight on crystallization. We obtained molecular weight distributions $(Đ<1.20)$ that can be considered relatively narrow. Nuclear magnetic resonance spectra $\left({ }^{1} \mathrm{H}\right.$ NMR) and size exclusion chromatography (SEC) were employed to determine chemical structure and number-average molecular weights $\left(M_{\mathrm{n}}\right)$ of the materials and their block 
components. The samples under study are listed in Table 1, with composition of the blocks as subscript numbers and molecular weights of the entire diblock copolymer and terpolymer as superscript numbers. The melting points of each component determined by DSC previously ${ }^{33-36}$ are also given in Table 1.

Table 1. Molecular weight, composition and melting point data of the two samples employed in this work.

\begin{tabular}{|c|c|c|c|c|c|c|c|}
\hline Sample code & $\begin{array}{c}M_{n} \\
\left(\mathrm{gmol}^{-1}\right)\end{array}$ & $\begin{array}{c}T_{m}\left({ }^{\circ} \mathrm{C}\right) \\
\text { PEO }\end{array}$ & $\begin{array}{c}M_{n} \\
\left(\mathrm{gmol}^{-1}\right)\end{array}$ & $\begin{array}{c}T_{m}\left({ }^{\circ} \mathrm{C}\right) \\
\text { PCL }\end{array}$ & $\begin{array}{c}M_{n} \\
\left(\mathrm{gmol}^{-1}\right)\end{array}$ & $\begin{array}{l}T_{m}\left({ }^{\circ} \mathrm{C}\right) \\
\text { PLLA }\end{array}$ & $\begin{array}{c}Ð \\
\left(M_{w} / M_{n}\right)\end{array}$ \\
\hline & $\begin{array}{l}\text { PEO } \\
\text { block }\end{array}$ & block & $\begin{array}{l}\text { PCL } \\
\text { block }\end{array}$ & block & $\begin{array}{l}\text { PLLA } \\
\text { block }\end{array}$ & block & \\
\hline $\mathrm{PCL}_{43} \mathrm{PLLA}_{57}{ }^{15.4}$ & - & & 6600 & $52-55$ & 8800 & $134-139$ & 1.16 \\
\hline $\mathrm{PEO}_{23} \mathrm{PCL}_{34} \mathrm{PLLA}_{43}{ }^{19.9}$ & 4600 & $41-46^{*}$ & 6800 & $52-57 *$ & 8500 & $120-127^{*}$ & 1.18 \\
\hline
\end{tabular}

\section{Films Preparation.}

Film samples were prepared by spin coating. The polymers were dissolved in chloroform at room temperature to make $0.1 \%, 0.2 \%$ and $0.5 \% \mathrm{w} / \mathrm{w}$ solutions. The polymer solution was dropped onto a $1 \mathrm{~cm}^{2}$ Si wafer, which was previously cleaned and oxidized with UV light, and then, it was spin-coated. 


\section{Thermal Treatment to Induce Microphase Separation.}

All blocks within the diblock copolymer and triblock terpolymer under study were previously crystallized, employing a two-step crystallization method. To erase thermal history, the sample was first melted at $160{ }^{\circ} \mathrm{C}$ for 3 minutes. Then, to crystallize the PLLA block, it was cooled from the melt to $0{ }^{\circ} \mathrm{C}$ at $20^{\circ} \mathrm{C} \mathrm{min}{ }^{-1}$ and finally heated up to $81{ }^{\circ} \mathrm{C}$ at 60 ${ }^{\circ} \mathrm{C} \mathrm{min}^{-1}$. It was held at this temperature for $1 \mathrm{~h}$ crystallize the PLLA block to saturation. To crystallize the PCL block, the sample was then cooled to $49.5^{\circ} \mathrm{C}$ at $60{ }^{\circ} \mathrm{C} \min ^{-1}$ and held at this temperature for $1 \mathrm{~h}$. Finally, the sample was quenched to $25^{\circ} \mathrm{C}$ at $100{ }^{\circ} \mathrm{C}$ min $^{-1}$ (during this cooling, the PEO block can crystallize ${ }^{35}$, forming the thinnest lamellae of the three components). After the thermal treatment, the samples were examined by AFM and WAXS.

\section{Morphological Observations.}

The as-crystallized films were measured with a Dimension Icon microscope (Bruker, USA) equipped with heating accessories. The measurements were performed by ScanAsyst mode with Bruker probes (SCANASYST-AIR mode; $12 \mathrm{~nm}$ tip radius; 0.4N/m spring constant and $70 \mathrm{kHz}$ resonant frequency) to simultaneously image the microstructure topography and the mechanical properties (i.e., modulus).

To evaluate the microphase separation by atomic force microscopy (AFM) with an in situ heating stage, two particular in situ thermal protocols were used:

(i) One step heating method. The as-crystallized PCL43 $\mathrm{PLLA}_{57}{ }^{15.4}$ sample was heated from room temperature to $70{ }^{\circ} \mathrm{C}$ (a temperature above the melting point of the PCL block crystals, see Table 1 ) at $1{ }^{\circ} \mathrm{C} \min ^{-1}$ and held at this temperature for in situ characterization by AFM.

(ii) Two steps heating method. The as-crystallized $\mathrm{PEO}_{23} \mathrm{PCL}_{34} \mathrm{PLLA}_{43}{ }^{19.9}$ sample was 
heated from room temperature to $49{ }^{\circ} \mathrm{C}$ (to melt the PEO block crystals, see Table 1) at a heating rate of $1{ }^{\circ} \mathrm{C} \min ^{-1}$ and kept at this temperature for in situ AFM observations. Subsequently, it was heated up to $60{ }^{\circ} \mathrm{C}$ (to melt the PCL block crystals, see Table 1) also at $1{ }^{\circ} \mathrm{C} \cdot \mathrm{min}^{-1}$, and in situ AFM characterization was again performed to image the PLLA block crystals.

Wide Angle X-ray Scattering (WAXS) experiments.

WAXS experiments were carried out on films of the samples that were previously subjected to the crystallization protocol described earlier. The WAXS scattering patterns were acquired in situ to follow the evolution of the microphase separation as the samples were heated. The in situ WAXS measurements were conducted in the ALBA Synchrotron Radiation Facility (Cerdanyola del Valles, Barcelona, Spain) (beamline BL11-NCD). A Rayonix LX255-HS detector with a resolution of $1920 \times 5760$ pixels (pixel size: $40 \mu \mathrm{m}^{2}$ ) was employed to record the WAXS patterns on heating. Silver behenate and $\mathrm{Cr}_{2} \mathrm{O}_{3}$ standards were used to perform the calibration. The physical parameters were the following: tilt angle, $30^{\circ}$; effective scattering vector $q$ range, $8-22 \mathrm{~nm}^{-1}$; and sample-to-detector distance; 126.8 mm. The radiation source had a wavelength $(\lambda)$ of $0.9999 \AA$. The DAWN software was used to process the data and to produce intensity plots as a function of the scattering vector, $q$ ( $q$ $=2 \pi / \mathrm{d}=4 \pi \sin \theta / \lambda$ ). The temperature was controlled employing a Linkam Scientific Instruments THMS600 hot-stage with a liquid nitrogen cooling system. WAXS patterns were recorded on heating between 25 and $160{ }^{\circ} \mathrm{C}$. The heating rate was set at $5{ }^{\circ} \mathrm{C} \mathrm{min}^{-1}$. An acquisition time of $6 \mathrm{~s}$ was used for each pattern. Thus, the temperature resolution was 0.5 ${ }^{\circ} \mathrm{C}$. The WAXS patterns are presented between 1 and $2 \mathrm{~A}^{-1}$. In this range, most crystallographic reflections of the blocks species under study are observed. 


\section{RESULTS AND DISCUSSION}

Two samples were selected in this work, a $\mathrm{PEO}_{23} \mathrm{PCL}_{34} \mathrm{PLLA}_{43}{ }^{19.9}$ triblock terpolymer and its precursor, a PCL43 $\mathrm{PLLA}_{57}{ }^{15.4}$ diblock copolymer, as they can be regarded as model di and triblock copolymer and terpolymer samples with crystallizable blocks. Both samples are most probably melt miscible. SAXS observations in the melt showed no scattering, ${ }^{33,}{ }^{35}$, although the electron density between these blocks is quite similar. ${ }^{26}$ Upon cooling from the melt, both DSC and WAXS analysis showed that the two phases in the diblock and the three phases in the triblock crystallize sequentially, being the PLLA block the one that crystallizes first. Therefore, the crystallization of the PLLA block templates the morphology of both diblock and triblock. As a consequence, the successive crystallization of the other blocks takes place inside the interlamellar zones of the spherulites of PLLA. The result is a special spherulitic-type superstructure with intercalated lamellae of PEO and/or PCL self-assembled in a random fashion. After PLLA crystallization, the WAXS analysis demonstrated in the triblock terpolymer that PCL block is the second to crystallize and then, the PEO block. ${ }^{33}$

An image of this tri-lamellar morphology at room temperature observed by AFM has been published by us before. ${ }^{35}$ However, we could not observe the morphology by AFM as the sample was heated. In this work, on the other hand, by employing in situ hot-stage AFM, we are able to show the presence of the three clear, distinct phases and corroborate the DSC and WAXS observations. Samples were prepared by spin coating employing different solution concentrations to determine the best sample preparation conditions. The samples were subjected to a thermal protocol that includes two isothermal steps to induce each block crystallization until saturation. The crystallization time of each isothermal step was $1 \mathrm{~h}$, 
which is sufficient time to induce the thickest lamellar thickness at the conditions chosen and a saturated crystallization process (see Figure 1).

In the case of the triblock terpolymer (see Figure 1), first, the PLLA block was crystallized by cooling down the sample from $160{ }^{\circ} \mathrm{C}$ (in melt state) to $0{ }^{\circ} \mathrm{C}$ and then heating up until $81^{\circ} \mathrm{C}$. The PLLA block was isothermally crystallized at $81^{\circ} \mathrm{C}$ during $1 \mathrm{~h}$, while the other two blocks are in the molten state. Afterwards the sample was rapidly quenched until $49.5^{\circ} \mathrm{C}$, which is a temperature that induces the PCL block crystallization only. At that temperature, the PCL block was crystallized until saturation during $1 \mathrm{~h}$. Finally, the sample was rapidly quenched again down to $25^{\circ} \mathrm{C}$ and, during this last cooling ramp, the PEO block (present only in the triblock terpolymer) can crystallize (please see reference 35 for further details). The same protocol described in Figure 1 was used for the precursor diblock copolymer for comparison purposes.

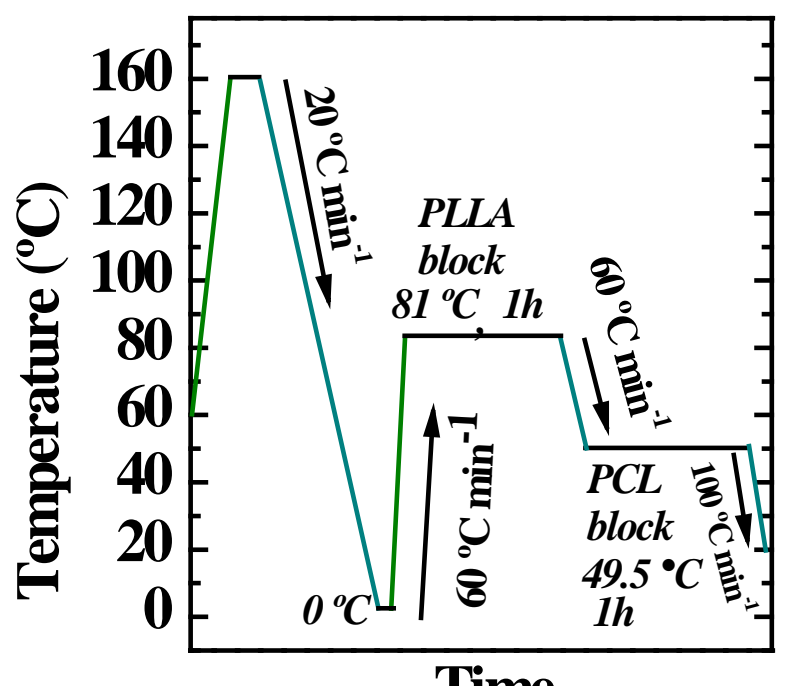

Time

Figure 1. Thermal crystallization protocol with two isothermal steps. 
The as-crystallized samples (following the protocol of Figure 1) were observed by AFM at $25{ }^{\circ} \mathrm{C}$ and during a subsequent heating scan employing a hot-stage. Two thermal protocols were employed (Figure 2): one step heating scan, in which AFM observations were made at 25 and $70{ }^{\circ} \mathrm{C}$, and a two-step heating scan, in which the observations were made at 25, 45, and $60{ }^{\circ} \mathrm{C}$. Figure 2 shows the thermal protocols applied during the in situ heating scans performed and the temperatures chosen.
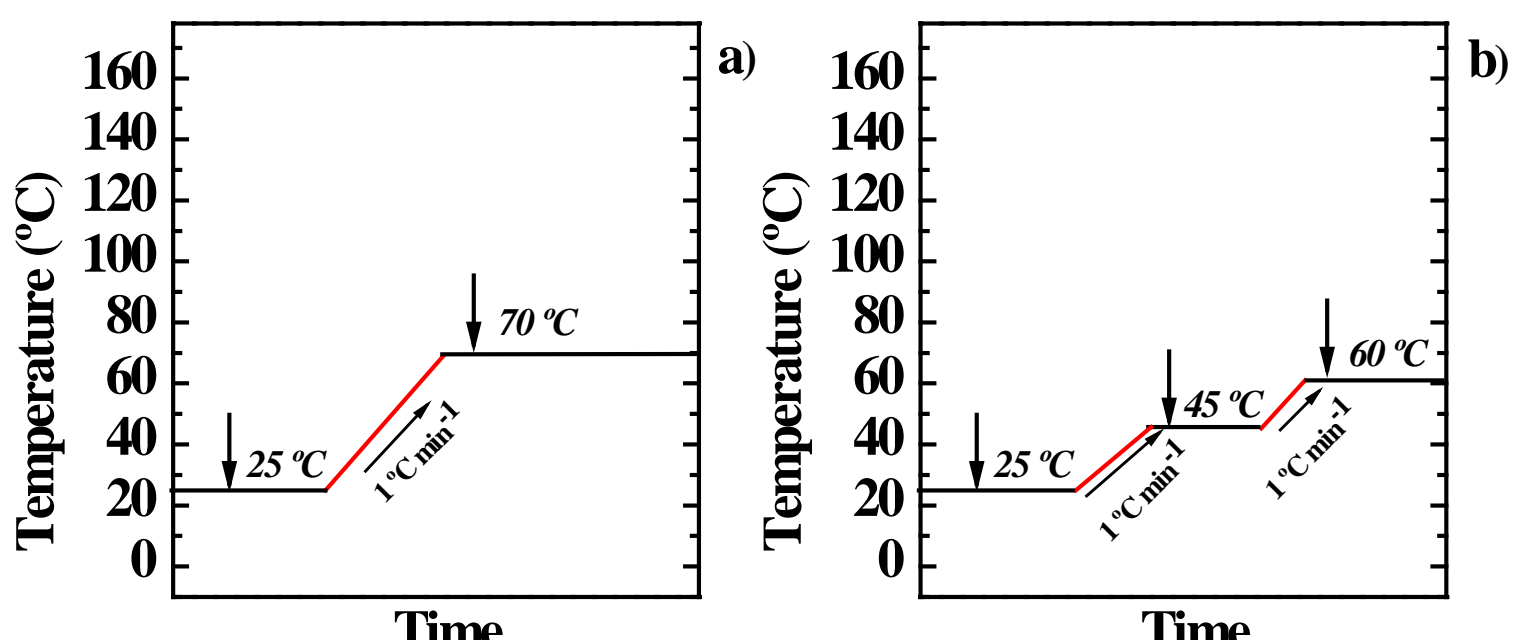

Figure 2. Thermal protocols applied to the crystallized samples (according to Figure 1) during heating: (a) One step heating protocol and (b) Two steps heating protocol.

\section{The alternated morphology of the PCL-b-PLLA diblock copolymer}

The nanoscale morphology of the $\mathrm{PCL}_{43} \mathrm{PLLA}_{57}{ }^{15.4}$ diblock copolymer was examined first. Figure 3 shows AFM height images of a sample prepared initially by spin-coating from solution but then subjected to the thermal crystallization protocol indicated in Figure 1. 
Figure 3a and $\mathrm{b}$ show the lamellar structure at $25{ }^{\circ} \mathrm{C}$ before applying the one-step heating scan described in Figure 2a. Most of the lamellae have grown in an edge-on fashion, allowing lamellar thickness measurements.

Figure 3a shows for the PCL $43{ }_{3} \mathrm{PLLA}_{57}{ }^{15.4}$ diblock copolymer sample, several packed lamellae that include alternated PCL and PLLA blocks lamellae. After careful observation and measurements of the AFM micrographs, two populations of different lamellar thickness were identified. One of them has an average thickness of $15 \mathrm{~nm}$, indicated with red dotted lines and the other, of $10 \mathrm{~nm}$, indicated with green dotted lines (Figure 3b). These values agree well with our previous report on the lamellar structure of these copolymers. ${ }^{35}$ In that report, ${ }^{35}$ the AFM observations were made by Multimode Scanning Probe Microscope (tapping mode) and using microfabricated silicon tips/cantilevers. We established that the thickest lamellae ( $15 \mathrm{~nm}$, red) should belong to the PLLA block because this block is the one that crystallizes first, while the thinnest lamellae $(10 \mathrm{~nm}$, green) should belong to the PCL block.

A WAXS experiment of the as-crystallized sample taken at $25{ }^{\circ} \mathrm{C}$ confirmed the presence of both types of crystals (see Figure 4a). The characteristic crystallographic planes of the $\alpha$-form of the PLLA crystals (110/200 and 113/203) can be observed, as well as, the 110, 111 and 200 reflections of the PCL crystals. However, and until now, it was not possible to assign without doubts to which block correspond each lamellar thickness.

Based on the different melting temperatures of each block (the PCL block melts at around $55^{\circ} \mathrm{C}$ and PLLA block at around $120{ }^{\circ} \mathrm{C}$, see Table 1 and our previous work ${ }^{35}$ ), the premise is that if the sample is heated until a temperature above the melting point of the PCL 
lamellae but sufficiently low to keep the PLLA phase crystallized, we should be able to see the disappearance of the PCL phase, while the PLLA lamellae will remain intact.
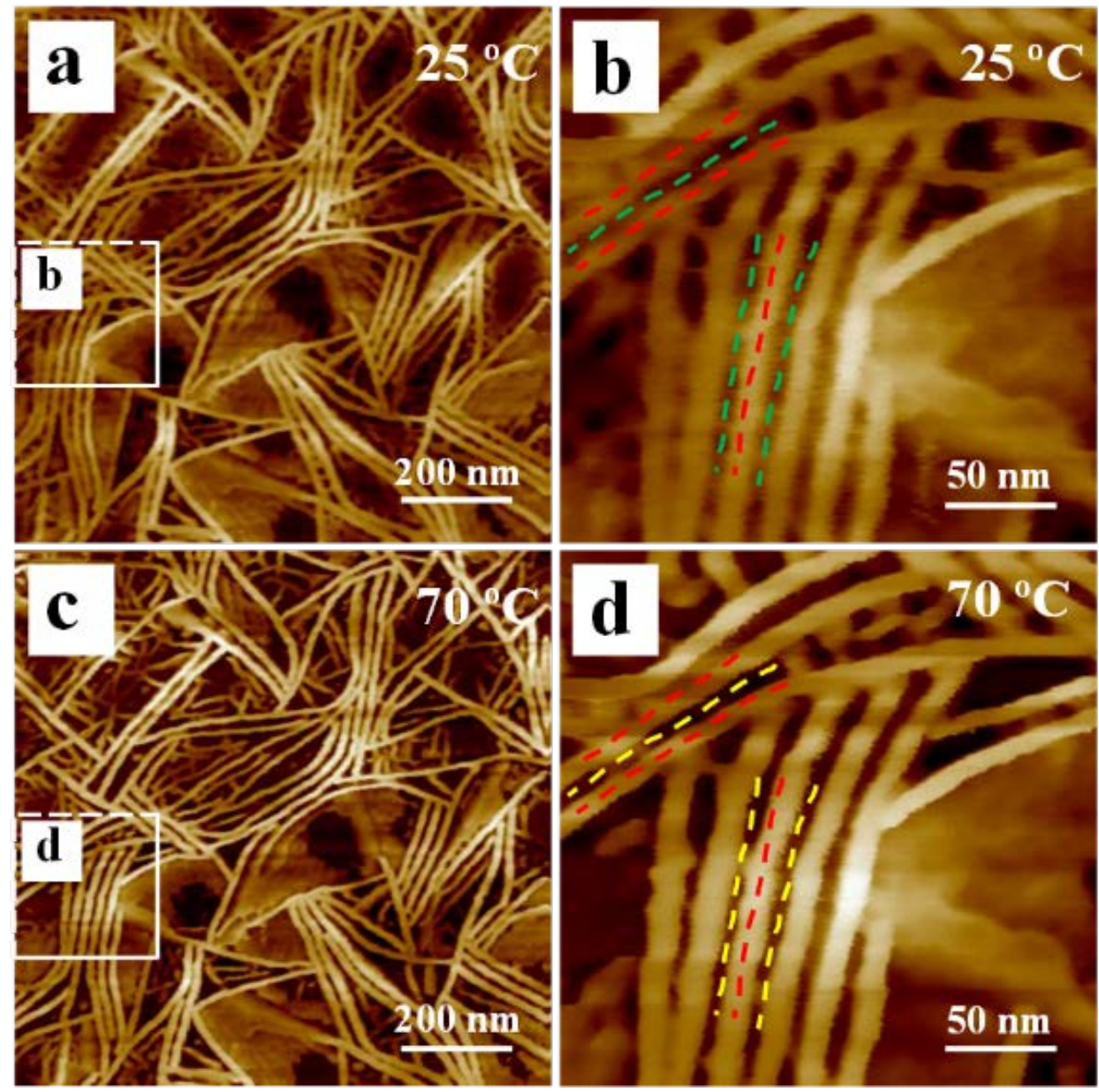

$42 \mathrm{~nm}$

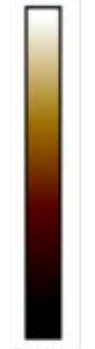

$0 \mathrm{~nm}$

Figure 3. AFM height images of a $\mathrm{PCL}_{43} \mathrm{PLLA}_{57}{ }^{15.4}$ sample prepared initially by spin-coating from a 0.2 wt\% solution and then subjected to the thermal crystallization protocol indicated in Figure 1. Images are given at $25^{\circ} \mathrm{C}(\mathrm{a}, \mathrm{b})$ and at $70{ }^{\circ} \mathrm{C}(\mathrm{c}, \mathrm{d})$ after the heating protocol described in Figure 2a. The dotted lines indicate PCL lamellae (green), PLLA lamellae (red), and molten PCL regions (yellow). 
Thus, to confirm the melting transitions of the PCL ${ }_{43} \mathrm{PLLA}_{57}{ }^{15.4}$ diblock copolymer, the as-crystallized sample (according to the protocol of Figure 1) was heated up, and simultaneous WAXS measurements were taken during heating.
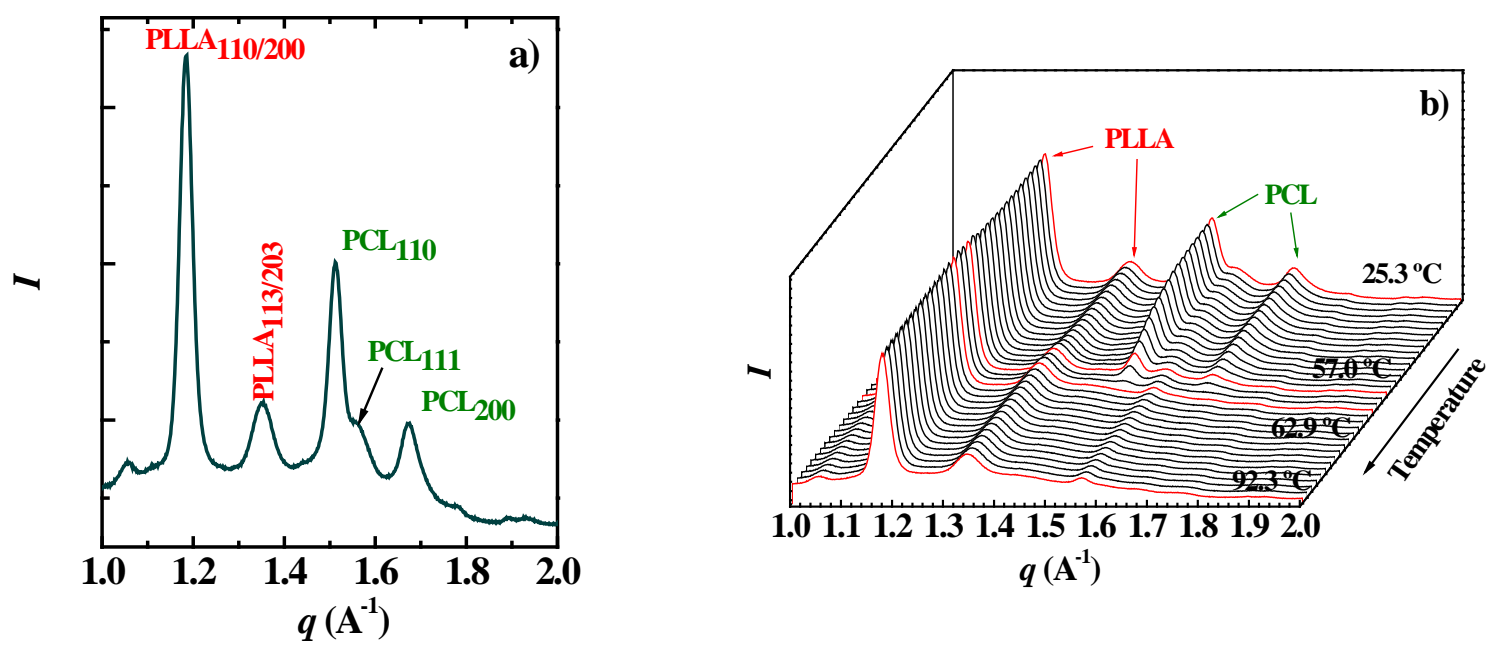

$q\left(\mathrm{~A}^{-1}\right)$

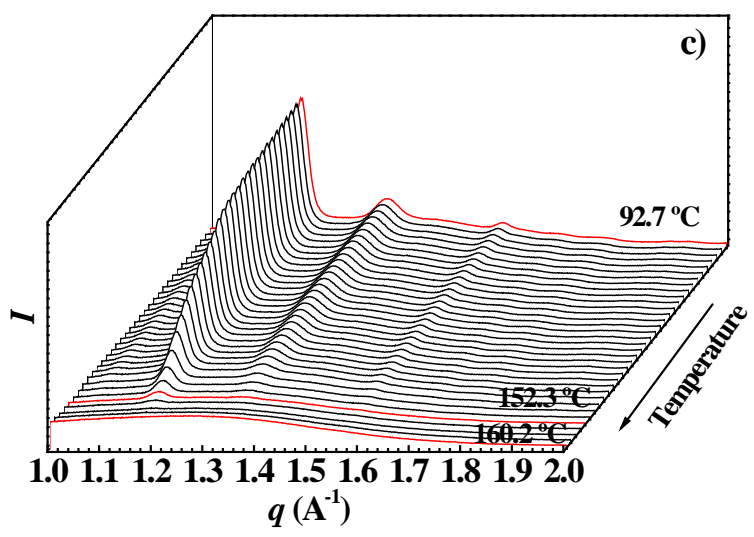

Figure 4. WAXS patterns of $\mathrm{PCL}_{43} \mathrm{PLLA}_{57}{ }^{15.4}$ diblock copolymer taken at $25{ }^{\circ} \mathrm{C}$, after crystallizing the sample in two isothermal steps (a). WAXS patterns evolution of $\mathrm{PCL}_{43} \mathrm{PLLA}_{57}{ }^{15.4}$ during subsequent heating between 25 and $92{ }^{\circ} \mathrm{C}$ (b) and between 92 and $160^{\circ} \mathrm{C}$ (c). 
It can be seen in Figure $4 \mathrm{~b}$ that at $25^{\circ} \mathrm{C}$, both PCL, and PLLA phases are present. But beyond $60{ }^{\circ} \mathrm{C}$, the reflections of the crystallographic planes of the PCL disappeared due to the melting of the crystals while the PLLA crystals remained unmolten. The PLLA phase melts completely at $160^{\circ} \mathrm{C}$ (see Figure 4c).

The as-crystallized sample was carefully heated $\left(1^{\circ} \mathrm{C} \cdot \mathrm{min}^{-1}\right)$ up to $70^{\circ} \mathrm{C}$ (protocol is shown in Figure 2a), and AFM observations at that temperature were performed. Unlike our previous report ${ }^{35}$, the AFM used on this occasion consists of a Dimension Icon microscope equipped with a hot-stage device that allows heating the sample in situ at a very low heating rate.

Figure $3 \mathrm{c}$ and $\mathrm{d}$ show the lamellar structure of the $\mathrm{PCL}_{43} \mathrm{PLLA}_{57}{ }^{15.4}$ diblock copolymer after heating the sample to $70{ }^{\circ} \mathrm{C}$ (a temperature above the melting point of the PCL block crystals). Comparing Figure $3 \mathrm{~b}\left(\right.$ at $25^{\circ} \mathrm{C}$ ) and Figure $3 \mathrm{~d}\left(\right.$ at $70{ }^{\circ} \mathrm{C}$ ), it is apparent that the smaller size lamellae (10 $\mathrm{nm}$, green) disappeared as a consequence of the melting of the PCL lamellae. Darker regions (indicated with yellow dot lines) can now be observed in between the bigger size lamellae that remained unchanged. These darker interlamellar regions correspond to the amorphous PCL phase that at $70{ }^{\circ} \mathrm{C}$ is molten. In fact, at this temperature, only PLLA reflections are observed in the WAXS spectrum (see Figure 4b). Therefore, the lamellar structure observed at $70{ }^{\circ} \mathrm{C}$ corresponds to PLLA lamellae, which are $15 \mathrm{~nm}$ in thickness. Thus, the PCL-b-PLLA diblock copolymer indeed exhibited a double crystalline morphology that includes lamellae of both PCL and PLLA at room temperature.

Cui et al. ${ }^{59}$ have previously reported the morphology of PCL-b-PLLA diblock copolymers employing a hot-stage AFM. However, the authors did not report a lamellar 
structure. On the contrary, at $25^{\circ} \mathrm{C}$, a wormlike co-continuous morphology of the blocks was proposed. However, the WAXS analysis demonstrated that only PCL crystals were present at room temperature. As the sample was heated, the PCL crystals melted, and the PLLA crystals started to grow, as confirmed by WAXS. The authors claimed that the wormlike structure was deformed due to the emergence of PLLA lamellae. However, a truly lamellar arrangement is not clear from the AFM images presented. Cooling from the melt to $80{ }^{\circ} \mathrm{C}$, a spherulitic-type texture seemed to emerge as a consequence of PLLA crystallization. Subsequent cooling to $27^{\circ} \mathrm{C}$ induced the PCL block crystallization, and the morphology was slightly changed. However, the crystalline structure is not clear ${ }^{59}$. These observations demonstrate the importance of a suitable sample preparation method and crystallization protocol to promote a clear lamellar crystalline morphology. Therefore, to our knowledge, this is the first time that in situ heating AFM experiments are employed to elucidate microphase separation and lamellar structure of different crystalline blocks in block copolymers.

Besides AFM techniques, the nanoscale lamellar morphology of PLLA- $b$-PCL diblock copolymers has also been observed in the literature by other microscopic techniques. In a previous report from Ho et al. ${ }^{62}$, the authors observed by TEM the morphology of crystals that have been crystallized from melt and grown epitaxially. Different crystallization temperatures and substrates were tested. At the crystallization temperatures chosen, only the PLLA block could crystallize, forming a branched crystalline lamellar structure. Flat-on PLLA lamellae were induced by the epitaxial crystallization on different subtracts, but the authors neglected the possible PCL crystallization at room temperature. Therefore, the 
authors presented a lamellar phase-separated structure that included both crystalline flat-on PLLA lamellae and alternating amorphous layers containing both PCL and PLLA chains.

Casas et al. ${ }^{63}$, on the other hand, reported solution grown single crystals of PCL- $b$ PLLA diblock copolymers that were observed by TEM. The PLLA block was crystallized first, forming truncated lozenge shape single crystals. Later, the PCL block crystallized forming fringed and small PCL crystals adjacent to the PLLA lamellae. Depending on the crystallization conditions, very complex crystalline morphologies were developed.

Further evidence of the alternated lamellar structure and phase assignment can be provided by the Dimension Icon Microscope employed in this work. This AFM device gives an image of the microstructural topography and at the same time determines the mechanical properties of the sample surface. Thus, properties such as adhesion and modulus can be scanned and mapped in the sample area.

The as-crystallized sample (crystallized as explained in Figure 1) consists of different phases that include PCL and PLLA crystalline phases and a mixed amorphous phase (containing both PCL and PLLA blocks chains). These three phases should exhibit a different mechanical response. For instance, the amorphous phase is softer than the crystalline one. And between PCL and PLLA, the mechanical features are also different. In general, tensile modulus values between 3000 and 4000 MPa have been reported for PLA, ${ }^{64}$, while PCL has been described as a softer material with modulus around $300-400 \mathrm{MPa}{ }^{65}$ Hence PLA is a more rigid material than PCL. Taking all this into consideration, the mechanical performance of the sample was determined employing the Dimension Icon Microscope AFM. We expected that some differences could be distinguished between the phases. Figure 5 shows 
the AFM modulus images of the $\mathrm{PCL}_{43} \mathrm{PLLA}_{57}{ }^{15.4}$ sample, initially prepared by spin-coating from solution, and then subjected to the thermal crystallization protocol indicated in Figure 1.
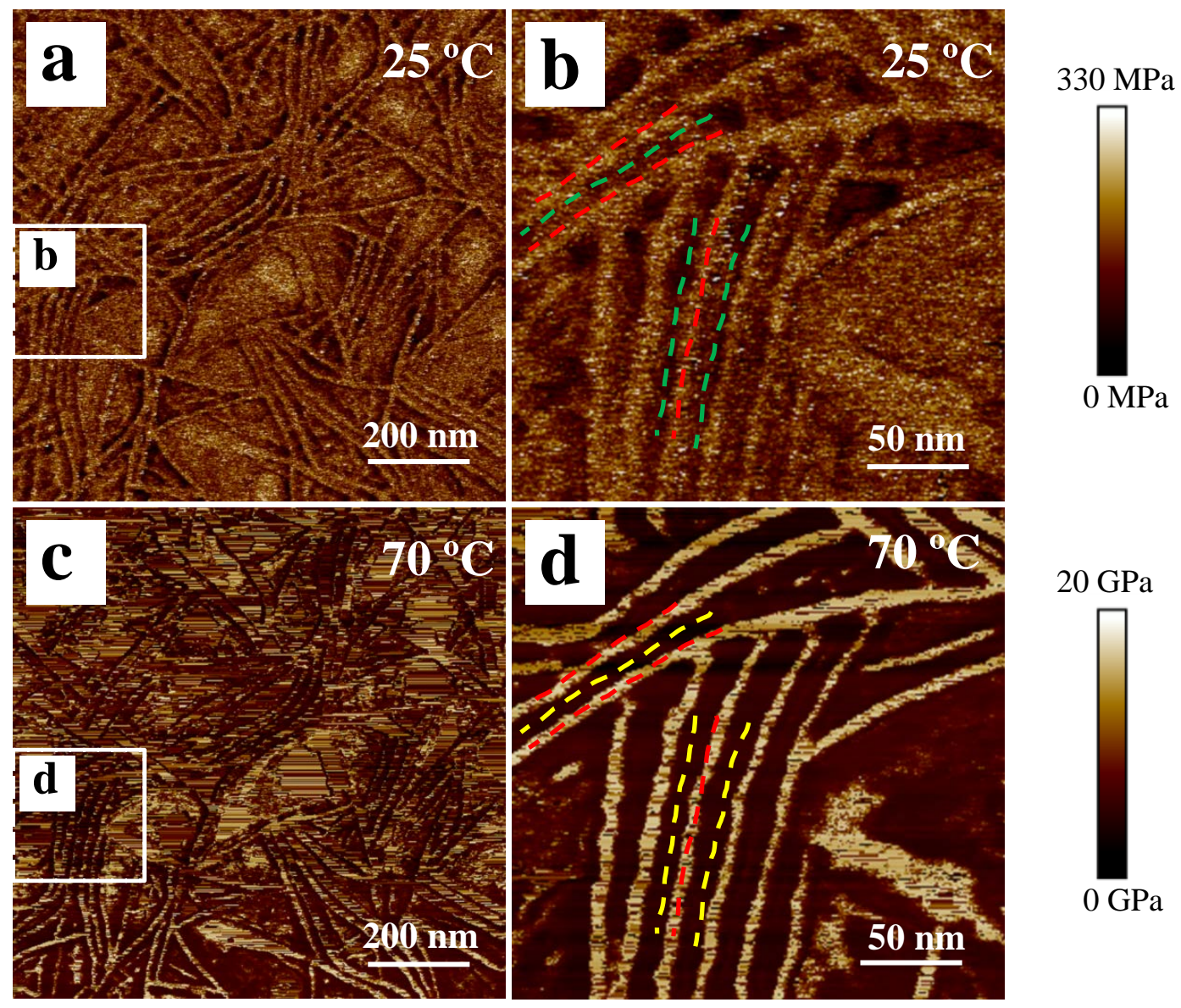

Figure 5. AFM modulus images of a $\mathrm{PCL}_{43} \mathrm{PLLA}_{57}{ }^{15.4}$ sample, originally prepared by spincoating from a $0.2 \mathrm{wt} \%$ solution, and then subjected to the thermal crystallization protocol indicated in Figure 1. Images are given at $25^{\circ} \mathrm{C}(\mathrm{a}, \mathrm{b})$, and at $70{ }^{\circ} \mathrm{C}(\mathrm{c}, \mathrm{d})$ after the heating protocol described in Figure 2a. The dotted lines indicate PCL lamellae (green), PLLA lamellae (red) and molten PCL regions (yellow). 
At $25^{\circ} \mathrm{C}$ (see Figure $5 \mathrm{a}$ and $\mathrm{b}$ ), it could be ${ }^{35}$ to elucidate the phases present, taking into consideration the modulus values only. If relative comparisons are made without taking into account the absolute values, a tentative assignment can be made as the brighter lamellae (of higher modulus) should correspond to the PLLA block (indicated with a red dotted lines) while less bright regions in between PLLA lamellae should be assigned to the PCL lamellae (green dotted line). However, this assignment is confirmed when the sample is heated to 70 ${ }^{\circ} \mathrm{C}$ (see Figure 5d). At that temperature, only the PLLA crystals remain, and the PCL lamellae have melted. Therefore, the very bright lamellae of high modulus are characteristic of the more rigid PLLA crystals. The PLLA lamellae remained unchanged and at the exact position as they were marked with red dotted lines in Figure 5b. On the contrary, the interlamellar PCL block regions appeared completely dark, indicating much lower values of elastic modulus than those in the PLLA block region. Being the amorphous phase a softer one, that observation is an indication of the melting of PCL lamellae and a clear evidence of the alternated lamellar structure, in which the PCL lamellae locates in an interdigitated fashion between the PLLA lamellae when it crystallizes within the previously formed PLLA spherulitic or axialitic templates.

\section{The interlamellar self-assembly of the PEO-b-PCL-b-PLLA triblock terpolymer}

Following the same approach employed for the diblock copolymer, the particular interlamellar arrangement of a tri-crystalline PEO- $b$-PCL- $b$-PLLA triblock terpolymer was studied. The sample selected, the $\mathrm{PEO}_{23} \mathrm{PCL}_{34} \mathrm{PLLA}_{43}{ }^{19.9}$, is a triblock terpolymer with the same PLLA and PCL block lengths as in the previously discussed diblock copolymer. The 
length of the PEO block is the smallest one (see Table 1). Thus, comparisons between the triblock terpolymer and the diblock copolymer (which is the precursor of the triblock terpolymer) can be made without any influence of molecular weights of the blocks under consideration.

The complexity of the nanoscale morphology of triblock terpolymers is high when the three blocks are able to crystallize. In our previous reports, ${ }^{33,35}$, we demonstrated by DSC and WAXS that the three blocks in $\mathrm{PEO}_{23} \mathrm{PCL}_{34} \mathrm{PLLA}_{43}$ are able to crystallize, and the SAXS analysis proved the melt miscibility. Upon cooling from melt, PLOM and WAXS experiments confirmed that PLLA crystallizes first, creating a spherulitic superstructural template. Then, the next block to crystallize is the PCL block, which must crystallize inside the interlamellar spaces of the PLLA spherulites (or axialites). The last block to crystallize is the PEO block, and it has no other option but to grow its lamellar crystals inside the available interlamellar spaces aside PCL and PLLA lamellae, as it is covalently bonded to the PCL block. The templated morphology did not change during the successive crystallization of the other PEO and PCL blocks, and only a slight change in the magnitude of the birefringence was observed. Therefore, after crystallization, the lamellae of the three blocks should coexist together in an alternated fashion. To confirm this hypothesis, we applied the crystallization protocol of Figure 1 to the $\mathrm{PEO}_{23} \mathrm{PCL}_{34} \mathrm{PLLA}_{43}{ }^{19.9}$ sample. The crystallization temperatures were chosen to be sufficiently high to induce the crystallization of each block separately and avoid the crystallization of the other two blocks. Hence, at $81^{\circ} \mathrm{C}$, the PEO and PCL blocks are molten, and only the PLLA block has the ability to crystallize. Then, at $49.5^{\circ} \mathrm{C}$, only the PCL block can crystallize (surrounded by the previously formed PLLA crystals), and the 
PEO block remains molten. Finally, the PEO block will crystallize during the last final quench treatment applied by cooling the sample to $25^{\circ} \mathrm{C}$ at $100{ }^{\circ} \mathrm{C} \cdot \mathrm{min}^{-1} \cdot 35$

In our previous report ${ }^{35}$, we were able to identify three different lamellar thicknesses by $\operatorname{AFM}(15,10$, and $7 \mathrm{~nm})$, and we hypothesized about their origin. The thickest lamellae of $15 \mathrm{~nm}$ should belong to the PLLA block because this value was similar to that of the PLLA block in the analogous diblock copolymer, and both blocks have the same molecular weight and were crystallized under identical conditions (i.e., at $81{ }^{\circ} \mathrm{C}$ for $1 \mathrm{~h}$ ). Then, the $10 \mathrm{~nm}$ lamellae were assigned to the PCL block due to the same reasons mentioned above: the PCL lamellae in the diblock copolymer were also around $10 \mathrm{~nm}$ thickness. Finally, the smallest lamellae should belong to the PEO block. But, to prove that, we had to run some WAXS measurements at room temperature and on heating to confirm the presence of PEO crystals. Specifically, the crystallographic planes in both the diblock copolymer and the triblock terpolymer were compared. Both PLLA and PEO reflections were observed. However, some reflections of PLLA and PEO crystals overlap (more details are $\mathrm{in}^{35}$ ). Therefore, after the WAXS experiment, the presence of the PEO crystals could only be indirectly confirmed.

In the present work, we take advantage of the AFM experiments employing a Dimension Icon microscope equipped with a hot-stage. The $\mathrm{PEO}_{23} \mathrm{PCL}_{34} \mathrm{PLLA}_{43}{ }^{19.9}$ sample was crystallized, as explained in Figure 1, and then it was in situ slowly heated following the thermal protocol described in Figure 2b. Then, AFM images were registered at different temperatures on heating. 

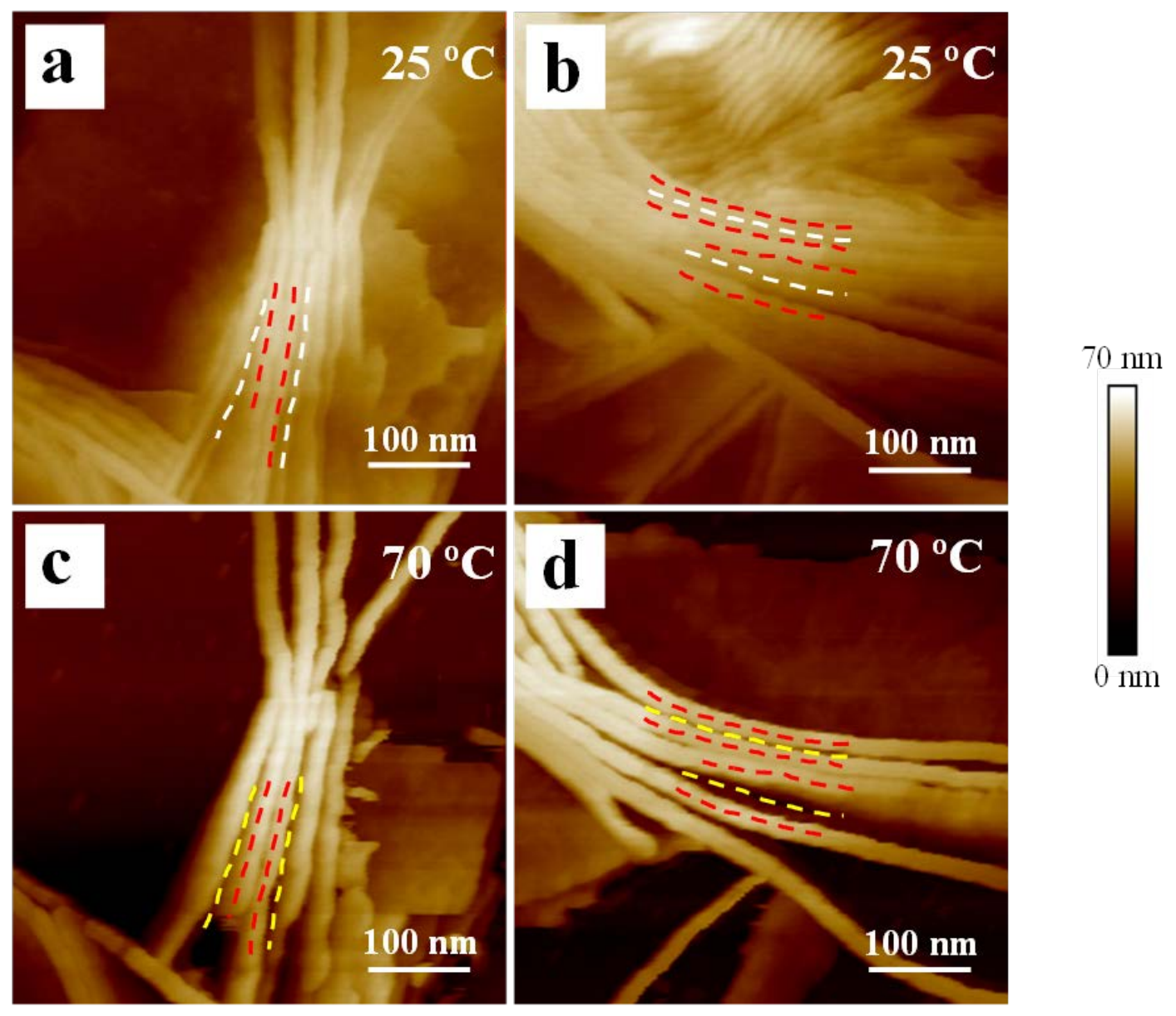

Figure 6. AFM height images of a $\mathrm{PEO}_{23} \mathrm{PCL}_{34} \mathrm{PLLA}_{43}{ }^{19.9}$ sample, initially prepared by spincoating from a $0.2 \mathrm{wt} \%$ solution and then subjected to the thermal crystallization protocol indicated in Figure 1. Images are given at $25^{\circ} \mathrm{C}(\mathrm{a}, \mathrm{b})$, and at $70{ }^{\circ} \mathrm{C}(\mathrm{c}, \mathrm{d})$ after the heating protocol described in Figure 2a. The dotted lines indicate PCL/PEO lamellae (white), PLLA lamellae (red) and molten PCL/PEO regions (yellow).

Figure 6 shows AFM height images of the triblock terpolymer sample prepared first by spin-coating, and then thermally treated according to Figure 1 . The aim is to identify the tri-lamellar structure of this multiphasic terpolymer. At $25{ }^{\circ} \mathrm{C}$, it can be seen that some 
lamellae grew edge-on, while others are slightly tilted. This lamellar arrangement should include lamellae of the PLLA, PCL and PEO crystals. The WAXS experiment at $25{ }^{\circ} \mathrm{C}$ revealed the crystallographic planes of the three blocks (see Figure 7a).

Detailed measurements of the lamellar thickness at $25{ }^{\circ} \mathrm{C}$ (see Figure 6a and b) revealed two populations of different lamellar thickness. The thickest one is $17 \mathrm{~nm}$ (red dotted line), and between these lamellae, another phase measures $11 \mathrm{~nm}$ (white dotted lines). Comparing these values with those of the diblock copolymer, the thickness values of the bigger size lamellae are close. Since the molecular weight of the PLLA blocks in both samples is similar and the crystallization conditions are the same, it is reasonable to assume that the $17 \mathrm{~nm}$ lamellae correspond to the PLLA block in the terpolymer. This value is also close to the $15 \mathrm{~nm}$ thickness that it was measured in the AFM images observed through the Multimode Scanning Probe Microscope that we previously reported. ${ }^{35}$ The small difference obeyed the fact that determining lamellar thickness by AFM is more sensitive to errors because it is a technique that scans a rather small surface area of the sample. Some limitations of determining lamellar thickness by AFM have been discussed before. ${ }^{66,67}$

Besides the $17 \mathrm{~nm}$ phase, the other one measured $11 \mathrm{~nm}$ (white dotted line). It is challenging to elucidate to which of the other two blocks correspond this phase. Increasing the temperature should melt one phase while keeping the other crystalline. It can be seen in Figure $7 \mathrm{~b}$ and $\mathrm{c}$ that beyond $60{ }^{\circ} \mathrm{C}$, only PLLA crystals remain. The effect of slowly increasing the temperature can be seen in the AFM images taken at $70{ }^{\circ} \mathrm{C}$. At this temperature, both PEO and PCL crystals are molten. It is clear that the phase marked with the white dotted line in Figure 6a and b disappeared in Figure 6c and d (signaled with a yellow dotted line). 

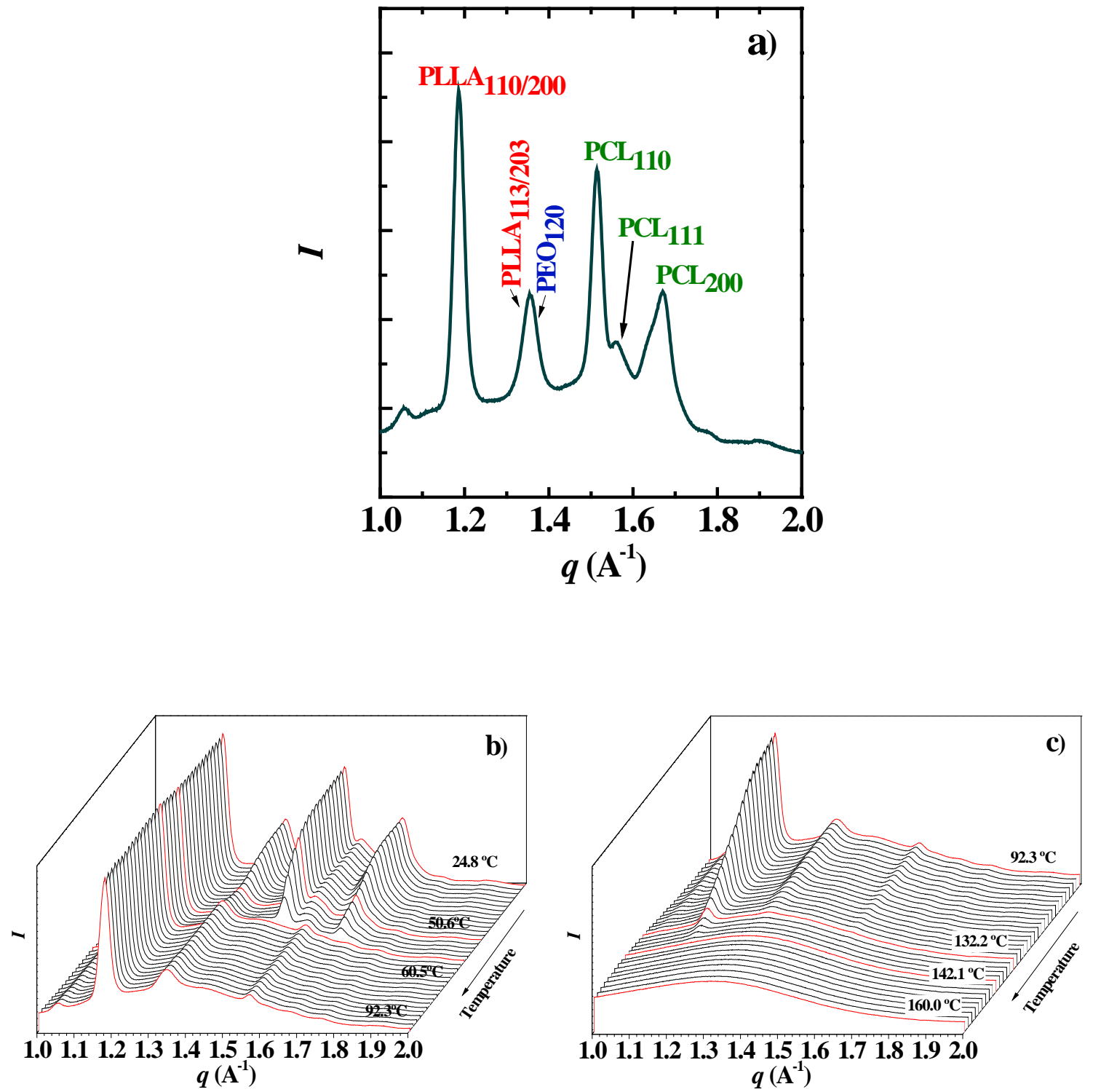

Figure 7. WAXS patterns of $\mathrm{PEO}_{23} \mathrm{PCL}_{34} \mathrm{PLLA}_{43}{ }^{19.9}$ diblock copolymer taken at $25{ }^{\circ} \mathrm{C}$, after crystallizing the sample in two isothermal steps (a). WAXS patterns evolution of $\mathrm{PEO}_{23} \mathrm{PCL}_{34} \mathrm{PLLA}_{43}{ }^{19.9}$ during subsequent heating between 25 and $92{ }^{\circ} \mathrm{C}$ (b) and between 92 and $160{ }^{\circ} \mathrm{C}(\mathrm{c})$. 

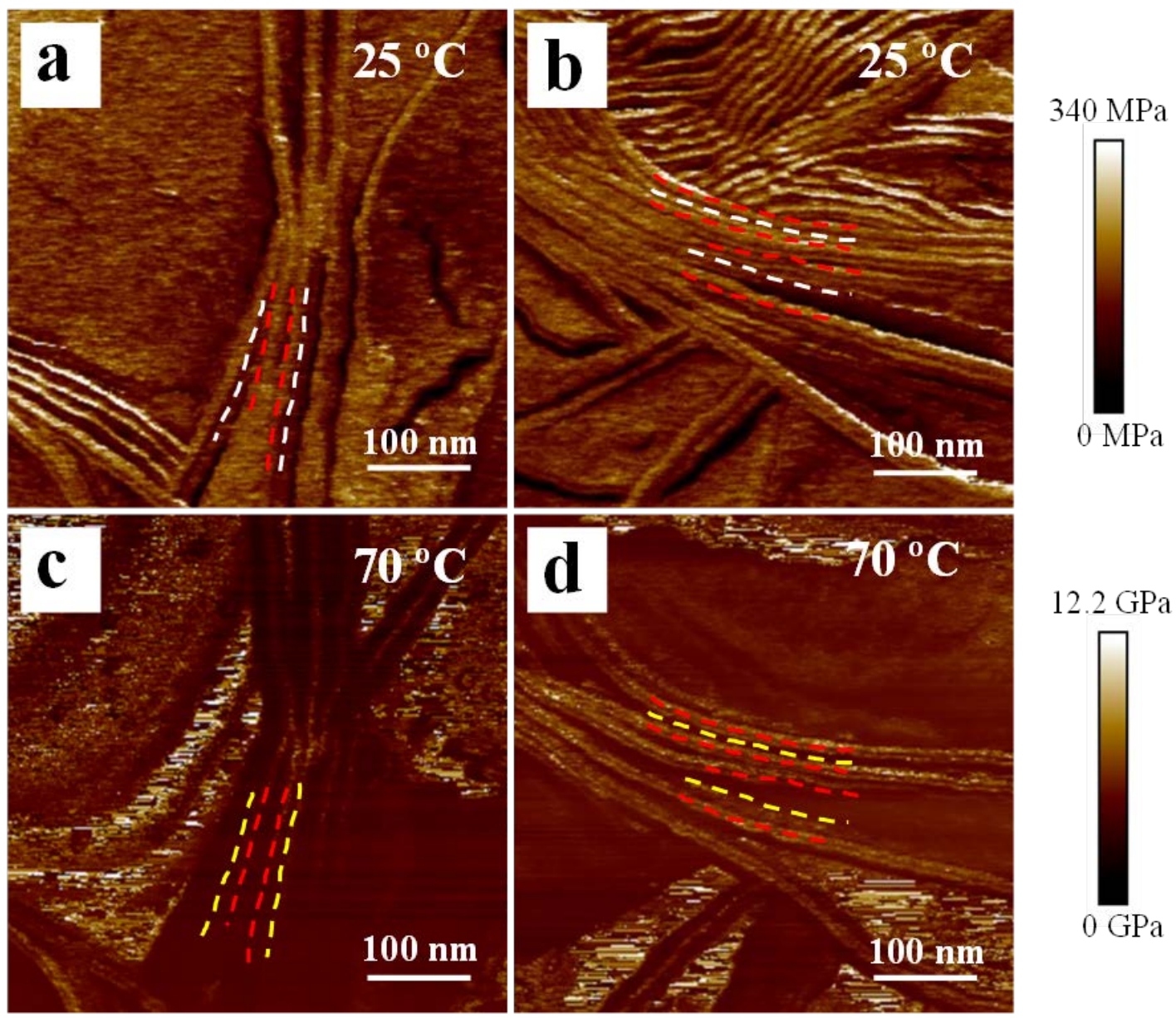

Figure 8. AFM modulus images of a $\mathrm{PEO}_{23} \mathrm{PCL}_{34} \mathrm{PLLA}_{43}{ }^{19.9}$ sample, initially prepared by spin-coating from a $0.2 \mathrm{wt} \%$ solution, and then subjected to the thermal crystallization protocol indicated in Figure 1. Images are given at $25^{\circ} \mathrm{C}(\mathrm{a}, \mathrm{b})$, and at $70{ }^{\circ} \mathrm{C}(\mathrm{c}, \mathrm{d})$ after the heating protocol described in Figure 2a. The dotted lines indicate PCL/PEO lamellae (white), PLLA lamellae (red) and molten PCL/PEO regions (yellow).

Therefore, the AFM images allow confirming that the thickest lamellae $(17 \mathrm{~nm}$, red dotted line) belong to the PLLA crystals since these are the only lamellae remaining at $70{ }^{\circ} \mathrm{C}$ (a temperature above the melting point of both PEO and PCL blocks). The AFM modulus 
images also confirmed this observation (see Figure 8). The darker interlamellar region inbetween the PLLA lamellae account for the softer amorphous phase resulting mainly from the molten PEO/PCL phase.

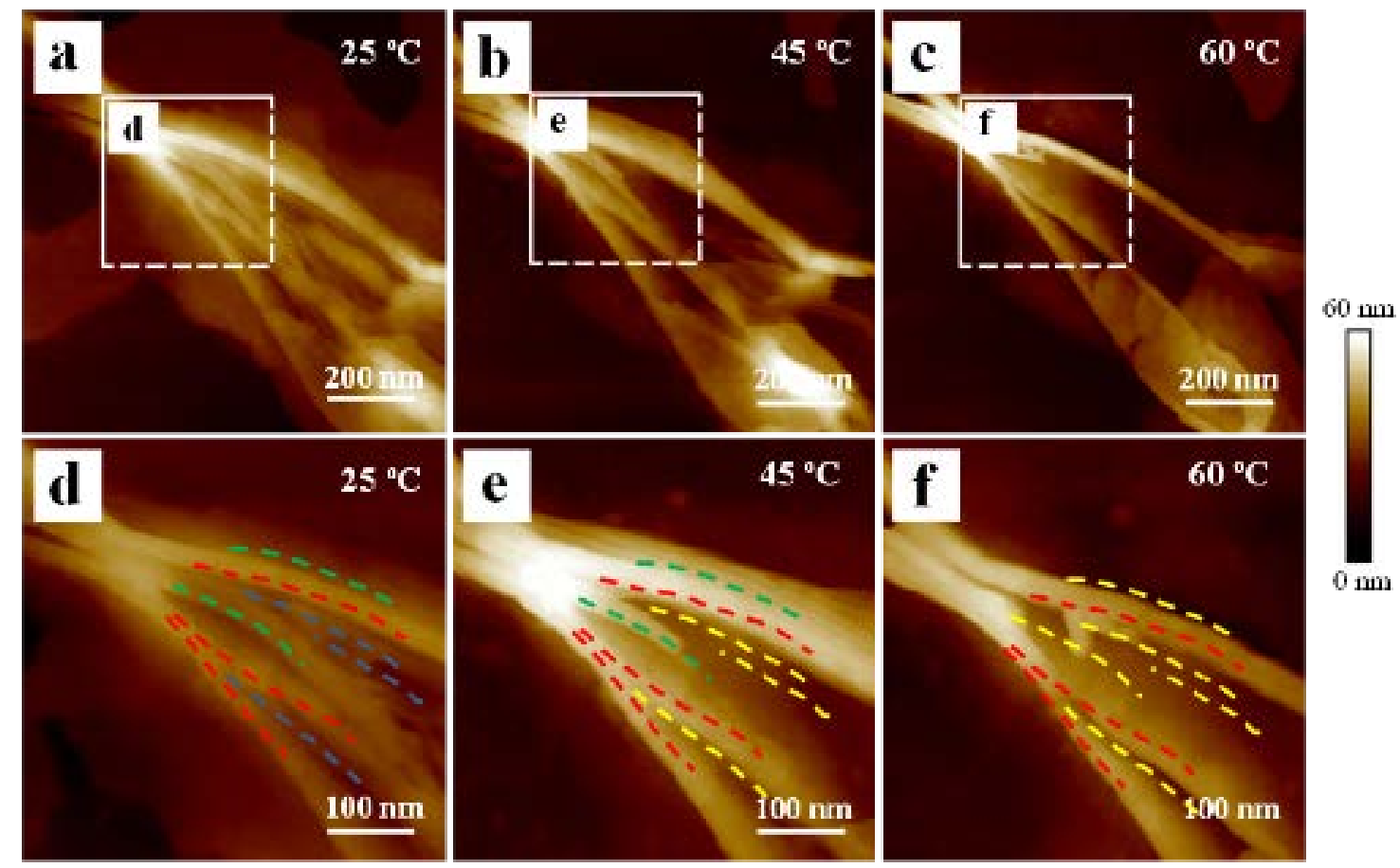

Figure 9. AFM height images of a $\mathrm{PEO}_{23} \mathrm{PCL}_{34} \mathrm{PLLA}_{43}{ }^{19.9}$ sample, initially prepared by spincoating from a $0.1 \mathrm{wt} \%$ solution, and then subjected to the thermal crystallization protocol indicated in Figure 1. Images are given at $25{ }^{\circ} \mathrm{C}(\mathrm{a}, \mathrm{b}), 45^{\circ} \mathrm{C}(\mathrm{b}, \mathrm{c})$ and $60{ }^{\circ} \mathrm{C}(\mathrm{d}$, e). The dotted lines indicate PEO lamellae (blue), PCL lamellae (green), PLLA lamellae (red), and the PEO/PCL melt region (yellow).

Nevertheless, trying to differentiate the PEO lamellae from the PCL lamellae from the images shown in Figure 8 for the $\mathrm{PEO}_{23} \mathrm{PCL}_{34} \mathrm{PLLA}_{43}{ }^{19.9}$ triblock terpolymer is more 
difficult than in the case of the diblock copolymer (Figure 6). Thus, to improve the quality of the AFM images, another sample solution of $0.5 \mathrm{wt} \%$ was prepared by spin-coating, and the film obtained melt crystallized as in Figure 1. Even though the sample solution was more concentrated, the multiphasic lamellar structure of the $0.5 \mathrm{wt} \%$ sample was not sufficiently clear in the AFM images taken with the Dimension Icon microscope (see Supplementary Information), even after melting the samples. Therefore, the solution concentration was diluted down to $0.1 \mathrm{wt} \%$, and the sample film was again crystallized following the same protocol described in Figure 1. After crystallization, the sample was slowly in situ heated, and AFM images were taken at 25,45 , and $60^{\circ} \mathrm{C}$, as described in Figure $2 \mathrm{~b}$. The sequence of AFM images on heating is presented in Figure 9 and Figure 10.

After employing a more diluted sample solution, the Dimension Icon AFM microscope confirms the multiphasic lamellar morphology that the authors hypothesized earlier. ${ }^{35}$ An extraordinary view of the tri-lamellar arrangement is given by the AFM height images taken at $25{ }^{\circ} \mathrm{C}$ (see Figure 9a). Some of the lamellae are edge-on, and other lamellae are slightly tilted. After a closer observation and exhaustive measurements, three populations of different lamellar thickness were identified: 18, 14, and $10 \mathrm{~nm}$ (see Figure 9d). These values are slightly higher than those that were previously reported: 15,10 , and $7 \mathrm{~nm} .{ }^{35}$ As was aforementioned, the small differences between AFM techniques obey their inherent sensitiveness to measurement errors.

What is relevant is the fact that a clear trilayered morphology is observed at $25^{\circ} \mathrm{C}$. In Figure 9d, the thickest lamellae (i.e., $18 \mathrm{~nm}$ ) are signaled with a red dotted line. While the intermediate size lamellae, with a green dotted line (i.e., $14 \mathrm{~nm}$ ), and the thinnest lamellae (i.e., $10 \mathrm{~nm}$ ) with a blue dotted. Thus, the premise is that each lamellae population might 
correspond to a different crystalline phase: whether PLLA, PCL, or PEO. This hypothesis was only indirectly proved through WAXS analysis measurements in a previous publication. ${ }^{35}$ In this report, we took advantage of the hot-stage AFM to determine to which phase belongs each lamella. Since each phase melts at different temperatures, we should be able to observe their disappearance as the sample is in situ heated.

Under standard crystallization conditions, the PEO and PCL blocks in the $\mathrm{PEO}_{23} \mathrm{PCL}_{34} \mathrm{PLLA}_{43}{ }^{19.9}$ triblock terpolymer melt at approximately 45 and $54{ }^{\circ} \mathrm{C}$, respectively, while the PLLA block does it at a much higher temperature, around $112-122{ }^{\circ} \mathrm{C}$ (see also Table 1). ${ }^{33}$ Figure $9 \mathrm{~b}$ shows the AFM height image of the sample after heating to $45{ }^{\circ} \mathrm{C}$. A closer observation (see Figure 9e) revealed the disappearance of the thinnest lamellae that were first marked with blue dotted lines. Since the PEO block melts at this temperature, the AFM height image proves undoubtedly that the thinnest lamellae belong to the PEO crystals. A yellow dotted line marks the position where the PEO lamellae were.

Further heating the sample up to $60^{\circ} \mathrm{C}$ causes the melting of the PCL phase. The medium size lamellae, indicated with a green dotted line in Figure 9, have now disappeared while the thickest lamellae marked with the red dotted line remain (see Figures 9c and f). At that temperature, only the PLLA block is crystalline, while the other two are completely molten.

The AFM images upon sequential heating confirm that the $14 \mathrm{~nm}$ lamellae are PCL block lamellar crystals, and the $18 \mathrm{~nm}$ lamellae correspond to the PLLA block. The exact position of where PCL lamellae were has been marked with yellow dotted lines in Figure 9f, while the lamellae that remain correspond to the PLLA block. The trilayered morphology has 
been confirmed, and the $\mathrm{PEO}_{23} \mathrm{PCL}_{34} \mathrm{PLLA}_{43}{ }^{19.9}$ triblock terpolymer can be prepared as a truly multi-crystalline system, provided suitable crystallization conditions are applied.

Although less clear, the multiphasic nanoscale morphology was also observed through AFM modulus images. Figure 10 shows the lamellar structure of the $\mathrm{PEO}_{23} \mathrm{PCL}_{34} \mathrm{PLLA}_{43}$ triblock terpolymer at different temperatures. It could be difficult to elucidate each phase by taking into account the mechanical modulus only.

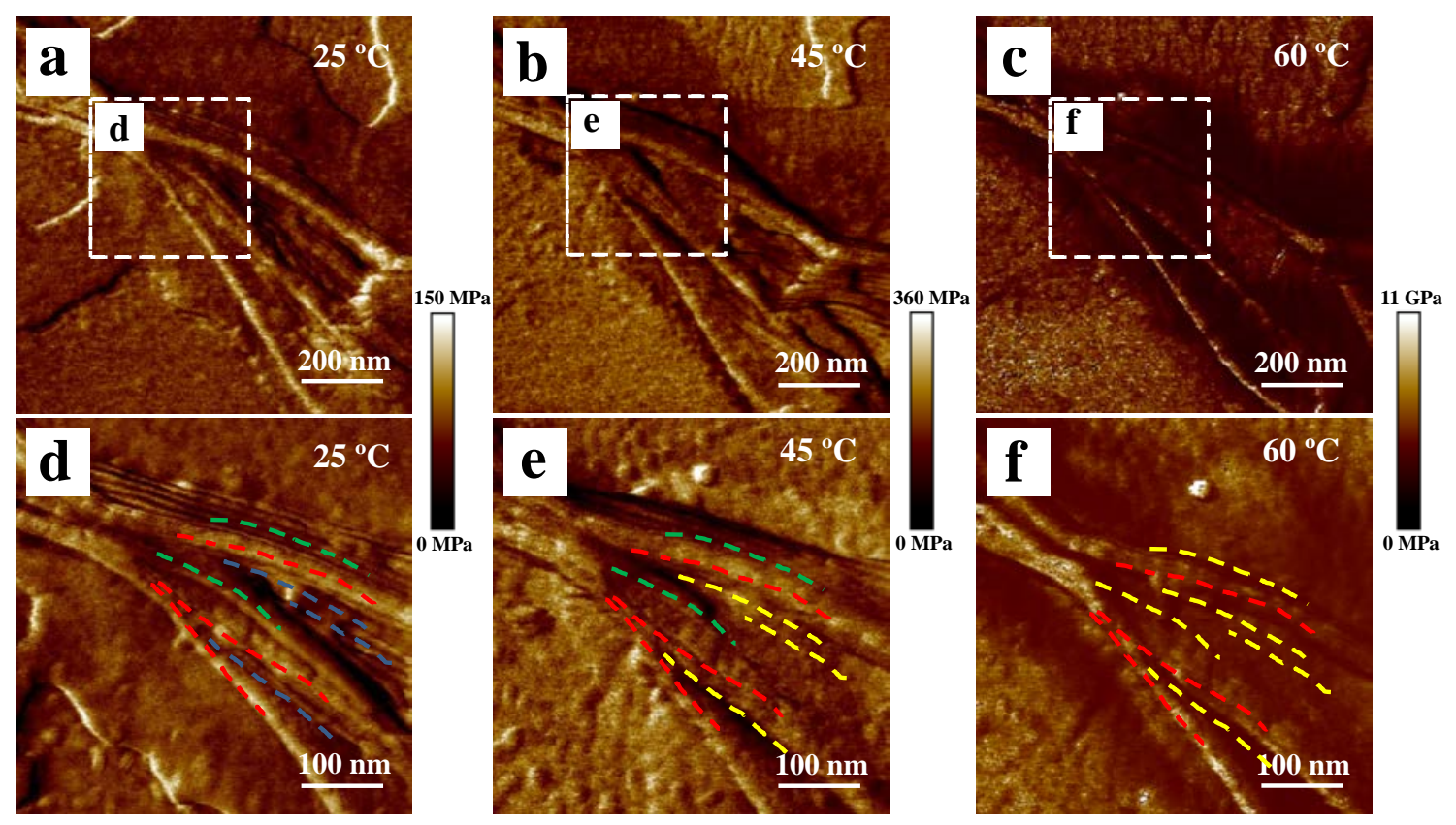

Figure 10. AFM modulus images of a $\mathrm{PEO}_{23} \mathrm{PCL}_{34} \mathrm{PLLA}_{43}{ }^{19.9}$ sample, originally prepared by spin-coating from a $0.1 \mathrm{wt} \%$ solution, and then subjected to the thermal crystallization protocol indicated in Figure 1. Images are given at $25^{\circ} \mathrm{C}(\mathrm{a}, \mathrm{b}), 45^{\circ} \mathrm{C}(\mathrm{b}, \mathrm{c})$ and $60^{\circ} \mathrm{C}(\mathrm{d}, \mathrm{e})$. The dotted lines indicate PEO lamellae (blue), PCL lamellae (green), PLLA lamellae (red), and the PEO/PCL melt region (yellow). 
Even though the PLLA is a more rigid material, while the PCL and PEO are softer polymers, it is complicated to differentiate them in the AFM modulus images at $25{ }^{\circ} \mathrm{C}$ (see Figure 10a and d). The reason is probably that PCL and PEO have comparable mechanical properties. ${ }^{65,68}$ However, as the sample is heated to 45 and $60{ }^{\circ} \mathrm{C}$, some lamellae disappeared while others remained brighter. Since the amorphous phase is a softer one, the mechanical modulus is lower as a result of the melting of the PCL and PEO crystals. Therefore, at $60{ }^{\circ} \mathrm{C}$, only the rigid-higher modulus crystalline phase of the PLLA block is observed, surrounded by a darker area (signaled with yellow dotted lines) that correspond to the melt mixed PCL/PEO phase.

Besides elucidating the nanoscale morphology, the hot-stage AFM technique could also be useful to provide some clarity in the fashion the lamellae arrange. In our previous publication, ${ }^{35}$ an exhaustive theoretical analysis of SAXS characterization upon heating suggested that a lamella of either PEO or PCL randomly inserts between two adjacent PLLA lamellae. This premise was proposed after modeling SAXS theoretical curves considering a one-dimensional structural model. At temperatures beyond the melting of the PCL and PEO blocks, the system can be described as a two-phase model. After several simulations, the best fit to the experimental SAXS curve was when only one lamella (of PEO or PCL ) fits into the interlamellar spaces of the PLLA crystals. However, the nanoscale structure observed in Figure 9a and d seems contrary to this statement. In Figure 9d, it can be observed that some lamellae of both PCL and PEO (green and blue dotted lines) intercalate between two lamellae of PLLA (red dotted lines). But, it is also possible to observe only one lamella of PEO (blue dotted line) in between two lamellae of PLLA. 
Despite this observation, it is essential to understand that the SAXS analysis is a broader range technique that can assess the structural features of the system in bulk. On the contrary, the AFM technique is a surface characterization tool that scans a very particular area of the sample surface and, therefore, might not represent the entire sample. What is significant is that the Dimension Icon AFM microscope not only showed to some extent the

random lamellar arrangement proposed earlier, ${ }^{35}$ but in addition, it gives clear evidence of the multi-crystalline morphology of triple crystalline PEO-b-PCL-b-PLLA triblock terpolymers. To our knowledge, this is the first time that hot-stage AFM is used to provide an accurate description of the nanoscale morphology of $\mathrm{ABC}$ type triblock terpolymer with three crystalline phases.

\section{CONCLUSIONS}

The hot-stage atomic force microscopy (AFM) has been used to accurately elucidate the tri-crystalline tri-layered morphology of a PEO-b-PCL-b-PLLA triblock terpolymer for the first time. This ABC type triblock terpolymer is melt miscible, and the three blocks can crystallize sequentially upon cooling from the melt. A two-step crystallization protocol was applied to the PEO-b-PCL-b-PLLA triblock terpolymer and to an analogous PCL- $b$-PLLA diblock copolymer for comparison purposes. Clear lamellar self-assembly, including lamellae of different lamellar thickness, was observed by AFM at room temperature. The WAXS analysis confirmed the presence of lamellae of each block. However, identifying each lamella in the terpolymer is not as straightforward. Considering the differences between the melting temperatures of the blocks, the sequential melting of the PEO-b-PCL- $b$-PLLA 
sample in the hot-stage allowed undoubtedly assigning the three crystalline lamellar thickness populations observed in the AFM images. The thickest lamellae belong to the PLLA block, the medium size lamellae to the PCL block, and the thinnest lamellae to the PEO block. The lamellar self-assembly includes lamellae of PLLA that intercalates randomly with lamellae of PCL, PEO, or both. The hot-stage AFM is a valuable technique to elucidate the nanoscale lamellar morphology of complex multi-crystalline systems.

\section{ACKNOWLEDGMENTS}

This work has received funding from the European Union's Horizon 2020 research and innovation program under the Marie Sklodowska-Curie grant agreement No 778092, from MINECO, project: MAT2017-83014-C2-1-P and from the Basque Government through grant IT1309-19. We are grateful to the National Science Foundation of China (nos. 51773182, 51973202), The Young Out-standing Teachers of the University in Henan Province (2019GGJS003). N.H. acknowledges the support of King Abdullah University of Science and Technology (KAUST).

\section{REFERENCES}

(1) Hamley, I. W., Block Copolymers. In Encyclopedia of Polymer Science and Technology, John Wiley \& Sons, Inc.: 2002; Vol. 1, pp 457-482.

(2) Huang, S.; Jiang, S. Structures and morphologies of biocompatible and biodegradable block copolymers. RSC Advances 2014, 4 (47), 24566-24583. 
(3) Nojima, S.; Marubayashi, H., Crystalline Morphology of Homopolymers and Block Copolymers. In Polymer Morphology: Principles, Characterization, and Processing, Guo, Q., Ed. John Wiley \& Sons, Inc: Hoboken, NJ, 2016; pp 165-180.

(4) Palacios, J. K.; Mugica, A.; Zubitur, M.; Müller, A. J., Crystallization and morphology of block copolymers and terpolymers with more than one crystallizable block. In Crystallization in Multiphase Polymer Systems, 2018; pp 123-180.

(5) Müller, A. J.; Arnal, M. L.; Lorenzo, A. T., Crystallization in Nano-Confined Polymeric Systems. In Handbook of Polymer Crystallization, Piorkowska, E., Rutledge, G. C., Eds. John Wiley and Sons: Hoboken, New Jersey, 2013; pp 347-372.

(6) Müller, A. J.; Arnal, M. L.; Balsamo, V. Crystallization in block copolymers with more than one crystallizable block. Lecture Notes in Physics 2007, 714, 229-259 DOI: 10.1007/3540-47307-6_13.

(7) Müller, A. J.; Balsamo, V.; Arnal, M. L. Nucleation and crystallization in diblock and triblock copolymers. Adv. Polym. Sci. 2005, 190, 1-63.

(8) Magonov, S. N. Atomic Force Microscopy in Analysis of Polymers. Encyclopedia of Analytical Chemistry 2006, 1-58 DOI: 10.1002/9780470027318.a2003.

(9) Schönherr, H.; Vancso, G. J., Scanning Force Microscopy of Polymers. Springer: Verlag Berlin Heidelberg, 2010.

(10) Schönherr, H., Imaging Polymer Morphology using Atomic Force Microscopy. In Polymer Morphology: Principles, Characterization, and Processing, Guo, Q., Ed. John Wiley \& Sons, Inc: Hoboken, NJ, 2016; pp 100-117. 
(11) Castillo, R. V.; Müller, A. J. Crystallization and morphology of biodegradable or biostable single and double crystalline block copolymers. Prog. Polym. Sci. 2009, 34 (6), 516-560.

(12) He, W. N.; Xu, J. T. Crystallization assisted self-assembly of semicrystalline block copolymers. Prog. Polym. Sci. 2012, 37 (10), 1350-1400 DOI: 10.1016/j.progpolymsci.2012.05.002.

(13) Nakagawa, S.; Marubayashi, H.; Nojima, S. Crystallization of polymer chains confined in nanodomains. Eur. Polym. J. 2015, 70, 262-275 DOI: 10.1016/j.eurpolymj.2015.07.018.

(14) Loo, Y. L.; Register, R. A.; Ryan, A. J. Modes of crystallization in block copolymer microdomains: Breakout, templated, and confined. Macromolecules 2002, 35 (6), 2365-2374 DOI: 10.1021/ma011824j.

(15) Boschetti-De-Fierro, A.; Fierro, D.; Albuerne, J.; Funari, S. S.; Abetz, V. Thermal monitoring of morphology in triblock terpolymers with crystallizable blocks. J. Polym. Sci., Part B: Polym. Phys. 2007, 45 (23), 3197-3206 DOI: 10.1002/polb.21318.

(16) Müller, A. J.; Albuerne, J.; Marquez, L.; Raquez, J. M.; Degée, P.; Dubois, P.; Hobbs, J.; Hamley, I. W. Self-nucleation and crystallization kinetics of double crystalline poly(pdioxanone)-b-poly(e-caprolactone) diblock copolymers. Faraday Discuss. 2005, 128, 231252.

(17) Müller, A. J.; Albuerne, J.; Esteves, L. M.; Marquez, L.; Raquez, J. M.; Degée, P.; Dubois, P.; Collins, S.; Hamley, I. W. In Confinement effects on the crystallization kinetics and self-nucleation of double crystalline poly(p-dioxanone)-b-poly(e-caprolactone) diblock copolymers, Macromol. Sym., 2004; 2004; pp 369-382. 
(18) Albuerne, J.; Márquez, L.; Müller, A. J.; Raquez, J. M.; Degée, P.; Dubois, P.; Castelletto, V.; Hamley, I. W. Nucleation and crystallization in double crystalline poly(pdioxanone)-b-poly(E-caprolactone) diblock copolymers. Macromolecules 2003, 36 (5), 1633-1644.

(19) Arnal, M. L.; Balsamo, V.; López-Carrasquero, F.; Contreras, J.; Carrillo, M.; Schmalz, H.; Abetz, V.; Laredo, E.; Müller, A. J. Synthesis and characterization of polystyrene-bpoly(ethylene oxide)-b-poly( $\varepsilon$-caprolactone) block copolymers. Macromolecules 2001, 34 (23), 7973-7982 DOI: 10.1021/ma011058g.

(20) Balsamo, V.; Paolini, Y.; Ronca, G.; Müller, A. J. Crystallization of the polyethylene block in polystyrene-b-polyethylene-b-polycaprolactone triblock copolymers, 1: Selfnucleation behavior. Macromol. Chem. Phys. 2000, 201 (18), 2711-2720.

(21) Balsamo, V.; Müller, A. J.; Stadler, R. Antinucleation effect of the polyethylene block on the polycaprolactone block in ABC triblock copolymers. Macromolecules 1998, 31 (22), 7756-7763.

(22) Balsamo, V.; Müller, A. J.; Von Gyldenfeldt, F.; Stadler, R. Ternary ABC block copolymers based on one glassy and two crystallizable blocks: Polystyrene-blockpolyethylene-block-poly(e-caprolactone). Macromol. Chem. Phys. 1998, 199 (6), 10631070.

(23) Arnal, M. L.; Boissé, S.; Müller, A. J.; Meyer, F.; Raquez, J. M.; Dubois, P.; Prud'Homme, R. E. Interplay between poly(ethylene oxide) and poly(l-lactide) blocks during diblock copolymer crystallization. CrystEngComm 2016, 18 (20), 3635-3649 DOI: 10.1039/c6ce00330c. 
(24) Huang, S.; Jiang, S.; An, L.; Chen, X. Crystallization and morphology of poly(ethylene oxide-b-lactide) crystalline-crystalline diblock copolymers. J. Polym. Sci., Part B: Polym. Phys. 2008, 46 (13), 1400-1411.

(25) Li, Y.; Huang, H.; Wang, Z.; He, T. Tuning radial lamellar packing and orientation into diverse ring-banded spherulites: Effects of structural feature and crystallization condition. Macromolecules 2014, 47 (5), 1783-1792 DOI: 10.1021/ma402579d.

(26) Xue, F.; Chen, X.; An, L.; Funari, S. S.; Jiang, S. Soft nanoconfinement effects on the crystallization behavior of asymmetric poly(ethylene oxide)-block-poly( $\varepsilon$ - caprolactone) diblock copolymers. Polym. Int. 2012, 61 (6), 909-917 DOI: 10.1002/pi.4158.

(27) He, C.; Sun, J.; Ma, J.; Chen, X.; Jing, X. Composition dependence of the crystallization behavior and morphology of the poly(ethylene oxide)-poly(e-caprolactone) diblock copolymer. Biomacromolecules 2006, 7 (12), 3482-3489.

(28) Jiang, S.; He, C.; An, L.; Chen, X.; Jiang, B. Crystallization and ring-banded spherulite morphology of poly(ethylene oxide)-block-poly(e-caprolactone) diblock copolymer. Macromol. Chem. Phys. 2004, 205 (16), 2229-2234.

(29) Han, W.; Liao, X.; Yang, Q.; Li, G.; He, B.; Zhu, W.; Hao, Z. Crystallization and morphological transition of poly(l-lactide)-poly(?-caprolactone) diblock copolymers with different block length ratios. RSC Advances 2017, 7 (36), 22515-22523 DOI: 10.1039/c7ra03496b.

(30) Huang, S.; Li, H.; Jiang, S.; Chen, X.; An, L. Morphologies and structures in poly(llactide-b-ethylene oxide) copolymers determined by crystallization, microphase separation, and vitrification. Polym. Bull. 2011, 67 (5), 885-902. 
(31) Wang, J. L.; Dong, C. M. Synthesis, sequential crystallization and morphological evolution of well-defined star-shaped poly(e-caprolactone)-b-poly(L-lactide) block copolymer. Macromol. Chem. Phys. 2006, 207 (5), 554-562 DOI: 10.1002/macp.200500546. (32) Sun, J.; Hong, Z.; Yang, L.; Tang, Z.; Chen, X.; Jing, X. Study on crystalline morphology of poly(L-lactide)-poly(ethylene glycol) diblock copolymer. Polymer 2004, 45 (17), 5969-5977 DOI: 10.1016/j.polymer.2004.06.026.

(33) Palacios, J. K.; Mugica, A.; Zubitur, M.; Iturrospe, A.; Arbe, A.; Liu, G.; Wang, D.; Zhao, J.; Hadjichristidis, N.; Muller, A. J. Sequential crystallization and morphology of triple crystalline biodegradable PEO-b-PCL-b-PLLA triblock terpolymers. RSC Advances 2016, 6 (6), 4739-4750 DOI: 10.1039/c5ra25812j.

(34) Palacios, J. K.; Liu, G.; Wang, D.; Hadjichristidis, N.; Müller, A. J. Generating Triple Crystalline Superstructures in Melt Miscible PEO-b-PCL-b-PLLA Triblock Terpolymers by Controlling Thermal History and Sequential Crystallization. Macromol. Chem. Phys. 2019, 220 (20), 1900292 DOI: 10.1002/macp.201900292.

(35) Palacios, J. K.; Tercjak, A.; Liu, G.; Wang, D.; Zhao, J.; Hadjichristidis, N.; Müller, A. J. Trilayered Morphology of an ABC Triple Crystalline Triblock Terpolymer. Macromolecules 2017, 50 (18), 7268-7281 DOI: 10.1021/acs.macromol.7b01576.

(36) Palacios, J. K.; Zhao, J.; Hadjichristidis, N.; Müller, A. J. How the Complex Interplay between Different Blocks Determines the Isothermal Crystallization Kinetics of TripleCrystalline PEO-b-PCL-b-PLLA Triblock Terpolymers. Macromolecules 2017, 50 (24), 9683-9695 DOI: 10.1021/acs.macromol.7b02148. 
(37) Chiang, Y.-W.; Hu, Y.-Y.; Li, J.-N.; Huang, S.-H.; Kuo, S.-W. Trilayered Single Crystals with Epitaxial Growth in Poly(ethylene oxide)-block-poly(e-caprolactone)-blockpoly(l-lactide) Thin Films. Macromolecules 2015, 48 (23), 8526-8533.

(38) Liénard, R.; Zaldua, N.; Josse, T.; Winter, J. D.; Zubitur, M.; Mugica, A.; Iturrospe, A.; Arbe, A.; Coulembier, O.; Müller, A. J. Synthesis and Characterization of Double Crystalline Cyclic Diblock Copolymers of Poly(e-caprolactone) and Poly(l(d)-lactide) (c(PCL-bPL(D)LA)). $\quad$ Macromol. $\quad$ Rapid Commun. 2016, $37 \quad$ (20), 1676-1681 DOI: 10.1002/marc.201600309.

(39) Castillo, R. V.; Müller, A. J.; Raquez, J. M.; Dubois, P. Crystallization kinetics and morphology of biodegradable double crystalline PLLA- b -PCL diblock copolymers. Macromolecules 2010, 43 (9), 4149-4160.

(40) Hamley, I. W.; Parras, P.; Castelletto, V.; Castillo, R. V.; Müller, A. J.; Pollet, E.; Dubois, P.; Martin, C. M. Melt structure and its transformation by sequential crystallization of the two blocks within poly(L-lactide)-block-poly(e-caprolactone) double crystalline diblock copolymers. Macromol. Chem. Phys. 2006, 207 (11), 941-953.

(41) Cai, C.; Wang, L. U.; Donc, C. M. Synthesis, characterization, effect of architecture on crystallization, and spherulitic growth of poly(L-lactide)b-poly(ethylene oxide) copolymers with different branch arms. J. Polym. Sci., Part A: Polym. Chem. 2006, 44 (6), 2034-2044 DOI: $10.1002 /$ pola.21318.

(42) Hamley, I. W.; Castelletto, V.; Castillo, R. V.; Müller, A. J.; Martin, C. M.; Pollet, E.; Dubois, P. Crystallization in poly(L-lactide)-b-poly( $\varepsilon$-caprolactone) double crystalline diblock copolymers: A study using x-ray scattering, differential scanning calorimetry, and polarized optical microscopy. Macromolecules 2005, 38 (2), 463-472. 
(43) Shin, D.; Shin, K.; Aamer, K. A.; Tew, G. N.; Russell, T. P.; Lee, J. H.; Jho, J. Y. A morphological study of a semicrystalline poly(L-lactic acid-b-ethylene oxide-b-L-lactic acid) triblock copolymer. Macromolecules 2005, 38 (1), 104-109.

(44) Prilliman, S. G.; Kavanagh, A. M.; Scher, E. C.; Robertson, S. T.; Hwang, K. S.; Colvin, V. L. An in-situ hot stage for temperature-dependent tapping-mode ${ }^{\mathrm{TM}}$ atomic force microscopy. Rev. Sci. Instrum. 1998, 69 (9), 3245-3250 DOI: 10.1063/1.1149090.

(45) Zhang, R.; Zhuravlev, E.; Androsch, R.; Schick, C. Visualization of Polymer Crystallization by In Situ Combination of Atomic Force Microscopy and Fast Scanning Calorimetry. Polymers 2019, 11, 890.

(46) Zhang, B.; Chen, J.; Freyberg, P.; Reiter, R.; Mülhaupt, R.; Xu, J.; Reiter, G. HighTemperature Stability of Dewetting-Induced Thin Polyethylene Filaments. Macromolecules 2015, 48 (5), 1518-1523 DOI: 10.1021/ma502345p.

(47) Zhang, B.; Chen, J.; Baier, M. C.; Mecking, S.; Reiter, R.; Mulhaupt, R.; Reiter, G. Molecular-weight-dependent changes in morphology of solution-grown polyethylene single crystals. Macromol Rapid Commun 2015, 36 (2), 181-9 DOI: 10.1002/marc.201400433.

(48) Zhang, B.; Chen, J.; Zhang, H.; Baier, M. C.; Mecking, S.; Reiter, R.; Mülhaupt, R.; Reiter, G. Annealing-induced periodic patterns in solution grown polymer single crystals. RSC Advances 2015, 5 (17), 12974-12980 DOI: 10.1039/c4ra10563j.

(49) Qiao, C.-d.; Jiang, S.-c.; Ji, X.-l.; An, L.-j. In situ observation of melting and crystallization behaviors of poly( $\varepsilon$-caprolactone) ultra-thin films by AFM technique. Chin. J. Polym. Sci. 2013, 31 (9), 1321-1328 DOI: 10.1007/s10118-013-1333-5. 
(50) Mareau, V. H.; Prud'homme, R. E. In-Situ Hot Stage Atomic Force Microscopy Study of Poly(ع-caprolactone) Crystal Growth in Ultrathin Films. Macromolecules 2005, 38 (2), 398-408 DOI: 10.1021/ma0482359.

(51) Núñez, E.; Vancso, G. J.; Gedde, U. W. Morphology, crystallization, and melting of single crystals and thin films of star-branched polyesters with poly(-caprolactone) arms as revealed by atomic force microscopy. Journal of Macromolecular Science, Part B: Physics 2008, 47 (3), 589-607 DOI: 10.1080/00222340801955636.

(52) Beekmans, L. G. M.; Van der Meer, D. W.; Vancso, G. J. Crystal melting and its kinetics on poly(ethylene oxide) by in situ atomic force microscopy. Polymer 2002, 43 (6), $1887-$ 1895 DOI: 10.1016/S0032-3861(01)00748-0.

(53) Chen, E. Q.; Jing, A. J.; Weng, X.; Huang, P.; Lee, S. W.; Cheng, S. Z. D.; Hsiao, B. S.; Yeh, F. In situ observation of low molecular weight poly(ethylene oxide) crystal melting, recrystallization. Polymer 2003, 44 (19), 6051-6058 DOI: 10.1016/S0032-3861(03)00557-3. (54) Schönherr, H.; Frank, C. W. Ultrathin films of poly(ethylene oxides) on oxidized silicon. 2. In situ study of crystallization and melting by hot stage AFM. Macromolecules 2003, 36 (4), 1199-1208 DOI: 10.1021/ma020686a.

(55) Jiang, X.; Liu, X.; Liao, Q.; Wang, X.; Yan, D. D.; Huo, H.; Li, L.; Zhou, J. J. Probing interfacial properties using a poly(ethylene oxide) single crystal. Soft Matter 2014, 10 (18), 3238-3244 DOI: 10.1039/c3sm52975d.

(56) Wang, H.; Schultz, J. M.; Yan, S. Study of the morphology of poly(butylene succinate)/poly(ethylene oxide) blends using hot-stage atomic force microscopy. Polymer 2007, 48 (12), 3530-3539 DOI: 10.1016/j.polymer.2007.03.079. 
(57) Huang, Y.; Liu, X. B.; Zhang, H. L.; Zhu, D. S.; Sun, Y. J.; Yan, S. K.; Wang, J.; Chen, X. F.; Wan, X. H.; Chen, E. Q.; Zhou, Q. F. AFM study of crystallization and melting of a poly(ethylene oxide) diblock copolymer containing a tablet-like block of poly\{2,5-bis[(4methoxyphenyl) oxycarbonyl]styrene\} in ultrathin films. Polymer 2006, 47 (4), 1217-1225 DOI: 10.1016/j.polymer.2005.12.021.

(58) Zhang, P.; Wang, Z.; Huang, H.; He, T. Direct observation of the relief structure formation in the nearly symmetric poly(styrene)-block-poly(e-caprolactone) diblock copolymer thin film. Macromolecules 2012, 45 (22), 9139-9146 DOI: 10.1021/ma301531a. (59) Cui, D.; Tang, T.; Bi, W.; Cheng, J.; Chen, W.; Huang, B. Ring-opening polymerization and block copolymerization of L-lactide with divalent samarocene complex. J. Polym. Sci., Part A: Polym. Chem. 2003, 41 (17), 2667-2675 DOI: 10.1002/pola.10814.

(60) Alamri, H.; Zhao, J.; Pahovnik, D.; Hadjichristidis, N. Phosphazene-catalyzed ringopening polymerization of $\varepsilon$-caprolactone: influence of solvents and initiators. Polym. Chem. 2014, 5 (18), 5471-5478 DOI: 10.1039/c4py00493k.

(61) Zhao, J.; Pahovnik, D.; Gnanou, Y.; Hadjichristidis, N. Sequential polymerization of ethylene oxide, $\varepsilon$-caprolactone and l-lactide: A one-pot metal-free route to tri- and pentablock terpolymers. Polym. Chem. 2014, 5 (12), 3750-3753 DOI: 10.1039/c4py00429a.

(62) Ho, R. M.; Hsieh, P. Y.; Tseng, W. H.; Lin, C. C.; Huang, B. H.; Lotz, B. CrystallizationInduced Orientation for Microstructures of Poly(L-lactide)-b-poly( $\varepsilon$-caprolactone) Diblock Copolymers. Macromolecules 2003, 36 (24), 9085-9092 DOI: 10.1021/ma0347868.

(63) Casas, M. T.; Puiggalí, J.; Raquez, J. M.; Dubois, P.; Córdova, M. E.; Müller, A. J. Single crystals morphology of biodegradable double crystalline PLLA-b-PCL diblock copolymers. Polymer 2011, 52 (22), 5166-5177. 
(64) Fiori, S., Industrial Uses of PLA. In Poly(lactic acid) Science and Technology: Processing, Properties, Additives and Applications, Jimenez, A., Peltzer, M., Ruseckaite, R., Eds. The Royal Society of Chemistry: Cambridge, 2015; Vol. 12, pp 317-333.

(65) Cama, G.; Mogosanu, D. E.; Houben, A.; Dubruel, P., Synthetic biodegradable medical polyesters: Poly-e-caprolactone. In Science and Principles of Biodegradable and Bioresorbable Medical Polymers, Zhang, X., Ed. Woodhead Publishing: 2017; pp 79-105.

(66) Zhou, H.; Wilkes, G. L. Comparison of lamellar thickness and its distribution determined from d.s.c., SAXS, TEM and AFM for high-density polyethylene films having a stacked lamellar morphology. Polymer 1997, 38 (23), 5735-5747 DOI: http://dx.doi.org/10.1016/S0032-3861(97)00145-6.

(67) Trifonova, D.; Varga, J.; Vancso, G. J. AFM study of lamellar thickness distributions in high temperature melt-crystallization of $\beta$-polypropylene. Polym. Bull. 1998, 41 (3), 341-348 DOI: $10.1007 / \mathrm{s} 002890050372$.

(68) Back, D. M.; Schmitt, R. L., Ethylene Oxide Polymers. In Encyclopedia of Polymer Science and Technology, John Wiley \& Sons, Inc.: 2002. 
For Table of Contents only

PEO-b-PCL-b-PLLA Melting sequence

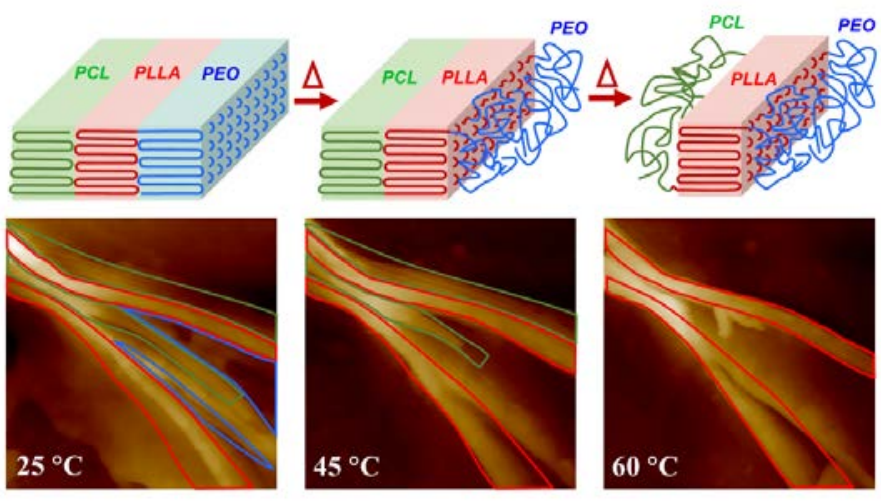

